\title{
APPROXIMATION OF FREE CONVOLUTIONS BY FREE INFINITELY DIVISIBLE LAWS.
}

\author{
G. P. CHISTYAKOV ${ }^{1,2}$ AND F. GÖTZE ${ }^{1,2}$
}

\begin{abstract}
Based on the method of subordinating functions we prove bounds for the minimal error of approximations of $n$-fold convolutions of probability measures by free infinitely divisible probability measures.
\end{abstract}

\section{INTRODUCTION}

In recent years a number of papers are investigating limit theorems for the free convolution of probability measures introduced by D. Voiculescu. The key concept of this definition is the notion of freeness, which can be interpreted as a kind of independence for non commutative random variables. As in classical probability where the concept of independence gives rise to the classical convolution, the concept of freeness leads to a binary operation on the probability measures on the real line, the free convolution. Many classical results in the theory of addition of independent random variables have their counterpart in this new theory, such as the law of large numbers, the central limit theorem, the Lévy-Khintchine formula and others. We refer to Voiculescu, Dykema and Nica [29], Hiai and Petz [17], Nica and Speicher [23] for a detailed introduction into these topics.

In classical probability theory Doeblin 15 showed that it is possible to construct independent identically distributed random variables $X_{1}, X_{2}, \ldots$ such that the distribution of the centered and normalized sum $b_{n_{k}}^{-1}\left(X_{1}+\cdots+X_{n_{k}}-a_{n_{k}}\right)$ does not converge to any non degenerate distribution, for any choice of the constants $a_{n}$ and $b_{n}$ and of sequences $n_{1}<n_{2}<\ldots$. In view of these examples Kolmogorov [19] suggested the class of infinitely divisible distributions for approximating the sequence $\left\{\mu^{n *}\right\}_{n=1}^{\infty}$ of convolutions of some distribution $\mu$ in some metric as $n \rightarrow \infty$. Starting with Prokhorov [24] and Kolmogorov [20] this problem had been investigated intensively, culminating in the seminal results by Arak and Zaitsev [2].

Date: October, 2020.

2020 Mathematics Subject Classification. Primary 46L53, Secondary 60E07.

Key words and phrases. Free random variables, Cauchy transforms, free convolutions, limit theorems.

1) Faculty of Mathematics, University of Bielefeld, Germany.

2) Research supported by CRC 1283. 
Due to the Bercovici-Pata parallelism between limits laws for free and classical additive convolution [8] the Doeblin results hold for free random variables and suggest that Kolmogorov approach is natural in free Probability Theory as well. Hence, we shall use this setup in free Probability Theory and study in this paper approximations of $n$-fold additive free convolutions of probability measures by additive free infinitely divisible probability measures.

The paper is organized as follows. In Section 2 we formulate and discuss the main results of the paper. In Section 3 we formulate auxiliary results. In Section 4 we obtain lower bounds in this class of approximations. We illustrate these approximation results in Section 5 with a list of examples of probability measures which allow for exponential small approximation errors whereas in Section 6 we construct probability measures which only allow polynomial small approximation errors.

\section{Results}

Denote by $\mathcal{M}$ the family of all Borel probability measures defined on the real line $\mathbb{R}$. On $\mathcal{M}$ define the associative composition laws denoted $*$ and $\boxplus$ as follows. For $\mu_{1}, \mu_{2} \in \mathcal{M}$ let a probability measure $\mu_{1} * \mu_{2}$ denote the classical convolution of $\mu_{1}$ and $\mu_{2}$. In probabilistic terms, $\mu_{1} * \mu_{2}$ is the probability distribution of $X+Y$, where $X$ and $Y$ are (commuting) independent random variables with distributions $\mu_{1}$ and $\mu_{2}$, respectively. A measure $\mu_{1} \boxplus \mu_{2}$ on the other hand denotes the free (additive) convolution of $\mu_{1}$ and $\mu_{2}$ introduced by Voiculescu [28] for compactly supported measures. Free convolution was extended by Maassen [22] to measures with finite variance and by Bercovici and Voiculescu [7] to the class $\mathcal{M}$. Thus, $\mu_{1} \boxplus \mu_{2}$ is the probability distribution of $X+Y$, where $X$ and $Y$ are free random variables with distributions $\mu_{1}$ and $\mu_{2}$, respectively.

Let $\Delta(\mu, \nu)$ be the Kolmogorov distance between probability measures $\mu$ and $\nu$, i.e.,

$$
\Delta(\mu, \nu)=\sup _{x \in \mathbb{R}}|\mu((-\infty, x))-\nu((-\infty, x))| .
$$

In 1955 Prokhorov [24] proved that for any $\mu \in \mathcal{M}$

$$
\Delta\left(\mu^{n *}, \mathbf{D}^{*}\right):=\inf _{\nu \in \mathbf{D}^{*}} \Delta\left(\mu^{n *}, \nu\right) \rightarrow 0, \quad n \rightarrow \infty,
$$

where $\mu^{n *}$ is the $n$-fold convolution of the probability measure $\mu$ and $\mathbf{D}^{*}$ is the set of classical infinitely divisible probability measures. Kolmogorov 20] noted that convergence in (2.1) to zero is uniform with respect to $\mu$ throughout the class $\mathcal{M}$. Work by a number of researches (a detailed history of the problem may be found in [2]) eventually proved upper and lower bounds for the function $\psi(n):=\sup _{\mu \in \mathcal{M}} \Delta\left(\mu^{n *}, \mathbf{D}^{*}\right)$. A final answer was given by Arak [3], [4], who proved the following bound: $c_{1} n^{-2 / 3} \leq \psi(n) \leq c_{2} n^{-2 / 3}$, where $c_{1}$ and $c_{2}$ are absolute positive constants. Extending Prohorov's results [24] in [10] the problem of determining the possible rate of decrease of $\Delta\left(\mu^{n *}, \mathbf{D}^{*}\right)$ to zero as $n \rightarrow \infty$ has been studied for probability measures $\mu \notin \mathbf{D}^{*}$.

Let $\mu \in \mathcal{M}$, denote $\mu^{n \boxplus}:=\mu \boxplus \cdots \boxplus \mu$ (n times). Recall that $\mu \in \mathcal{M}$ is $\boxplus$-infinitely divisible if, for every $n \in \mathbb{N}$, there exists $\mu_{n} \in \mathcal{M}$ such that $\mu=\mu_{n}^{n \boxplus}$. In the sequel we 
will write in this case that $\mu \in \mathbf{D}^{\boxplus}$. As in the classical case introduce the quantity

$$
\Delta\left(\mu^{n \boxplus}, \mathbf{D}^{\boxplus}\right):=\inf _{\nu \in \mathbf{D}^{\boxplus}} \Delta\left(\mu^{n \boxplus}, \nu\right)
$$

and raise the question of the behaviour of this quantity when $n \rightarrow \infty$.

Let $\mathbb{C}^{+}\left(\mathbb{C}^{-}\right)$denote the open upper (lower) half of the complex plane. For $\mu \in \mathcal{M}$, define its Cauchy transform by

$$
G_{\mu}(z)=\int_{-\infty}^{\infty} \frac{\mu(d t)}{z-t}, \quad z \in \mathbb{C}^{+} .
$$

It is clear that the quantity $c_{1}(\mu):=\Im\left(1 / G_{\mu}(i)\right)-1$ is positive if $\mu \neq \delta_{b}$ with $b \in \mathbb{R}$ and $\delta_{b}$ denoting a Dirac measure concentrated at the point $b$. In the sequel we denote by $c(\mu), c_{2}(\mu), c_{3}(\mu), \ldots$ positive constants depending on $\mu$ only. By $c(\mu)$ we denote constants in different (or even in the same) formulae.

We proved in [14] the following result.

Theorem 2.1. Let $\mu \in \mathcal{M}$ and $c_{1}(\mu)>0$. Then

$$
\Delta\left(\mu^{n \boxplus}, \mathbf{D}^{\boxplus}\right) \leq c(\mu)\left(\frac{1}{\sqrt{n}} \int_{\left[-N_{n}, N_{n}\right]}|u| \mu(d u)+\mu\left(\left\{\mathbb{R} \backslash\left[-N_{n} / 8, N_{n} / 8\right]\right\}\right), \quad n \in \mathbb{N},\right.
$$

where $N_{n}:=\sqrt{\sigma_{1}(\mu)(n-1)}$.

From this theorem the free analogue of Prokhorov's result (2.1) immediately follows.

\section{Corollary 2.2.}

$$
\Delta\left(\mu^{n \boxplus}, \mathbf{D}^{\boxplus}\right) \rightarrow 0, \quad \text { as } \quad n \rightarrow \infty .
$$

Denote by $\mathcal{M}_{d}, d \geq 0$, the set of probability measures such that $\beta_{d}(\mu):=\int_{\mathbb{R}}|x|^{d} \mu(d x)<$ $\infty$. We easily obtain from Theorem 2.1 the following upper bound.

Corollary 2.3. Let $\mu \in \mathcal{M}_{d}$ with some $d>0$. Then

$$
\Delta\left(\mu^{n \boxplus}, \mathbf{D}^{\boxplus}\right) \leq c(\mu) n^{-\min \{d, 1 / 2\}}, \quad n \in \mathbb{N} .
$$

Now we establish a possible rate of decrease to zero as $n \rightarrow \infty$ for $\mu \notin \mathbf{D}^{\boxplus}$. Denote $\mathbb{N}_{1}=\mathbb{N} \backslash\{1,2\}$.

Theorem 2.4. Let $\mu \in \mathcal{M}_{d}, d \in \mathbb{N}_{1}$, and $\mu \notin \mathbf{D}^{\boxplus}$. Then

$$
\liminf _{n \rightarrow \infty} \frac{\log \Delta\left(\mu^{n \boxplus}, \mathbf{D}^{\boxplus}\right)}{\sqrt{n}}>-\infty .
$$

Corollary 2.3 and Theorem 2.4 provide a two-sided estimate of the quantity $\Delta\left(\mu^{n \boxplus}, \mathbf{D}^{\boxplus}\right)$ for the probability measures $\mu \in \mathcal{M}_{d}, d \in \mathbb{N}_{1}$ we considered, and $\mu \notin \mathbf{D}^{\boxplus}$

$$
e^{-c_{2}(\mu) \sqrt{n}} \leq \Delta\left(\mu^{n \boxplus}, \mathbf{D}^{\boxplus}\right) \leq \frac{c_{3}(\mu)}{\sqrt{n}}, \quad n \in \mathbb{N} .
$$


Denote by $m_{s}(\mu):=\int_{\mathbb{R}} u^{s} \mu(d u), s=0,1, \ldots$, moments of the probability measure $\mu$. For $\mu \in \mathcal{M}_{d}$ with $d \geq 3$, we can improve the upper bound in (2.3). Let $\mu \in \mathcal{M}_{d}$ with $d \geq 3$ and $m_{1}(\mu)=0, m_{2}(\mu)=1$. Denote $\mu_{n}((-\infty, x)):=\mu((-\infty, x \sqrt{n})), x \in \mathbb{R}$.

Consider the family of $\boxplus$-infinitely divisible probability-measures $\left\{w_{a}: a \in \mathbb{R}\right\}$ with the Cauchy transform

$$
G_{w_{a}}(z)=\left(a+\frac{1}{2}\left(z-a+\sqrt{(z-a)^{2}-4}\right)\right)^{-1}, \quad z \in \mathbb{C} .
$$

These probability measures are a special case of the free centered (i.e. with mean zero) Meixner measures. In this formula the branch of the analytic square root should be chosen according to the condition $\Im z>0 \Longrightarrow \Im\left(1 / G_{w_{a}}(z)\right) \geq 0$.

In [13] it was proved for $\mu \in \mathcal{M}_{d}$ with $d \geq 3$ that there exists a positive absolute constant $c$ such that

$$
\Delta\left(\mu_{n}^{n \boxplus}, w_{a_{n}}\right) \leq c \frac{\beta_{d_{1}}(\mu)}{n^{\left(d_{1}-2\right) / 2}}, \quad n \in \mathbb{N},
$$

where $a_{n}:=m_{3}(\mu) / \sqrt{n}$ and $d_{1}:=\min \{d, 4\}$.

The estimate (2.5) shows that for $\mu \in \mathcal{M}_{d}$ with $d \geq 3$ the upper bound in (2.3) has the form

$$
\Delta\left(\mu^{n \boxplus}, \mathbf{D}^{\boxplus}\right) \leq c \frac{\beta_{d_{1}}(\mu)}{n^{\left(d_{1}-2\right) / 2}}, \quad n \in \mathbb{N} .
$$

In the sequel we denote by $\varphi(t ; \mu)$ the characteristic function of the probability measure $\mu$, i.e., $\varphi(t ; \mu)=\int_{\mathbb{R}} e^{i t x} \mu(d x), t \in \mathbb{R}$. We obtain the statement of Theorem 2.4 as a consequence of the next result.

Theorem 2.5. Let $\mu \in \mathcal{M}_{d}, d \in \mathbb{N}_{1}$, and the following relation is sutisfied:

$$
\liminf _{n \rightarrow \infty} \frac{\log \Delta\left(\mu^{n \boxplus}, \mathbf{D}^{\boxplus}\right)}{\sqrt{n}}=-\beta<0 .
$$

Then $\varphi(t ; \mu)=\varphi(t ; \rho), t \in\left[-\frac{\beta}{300 \sqrt{m_{2}(\mu)-m_{1}^{2}(\mu)}}, \frac{\beta}{300 \sqrt{m_{2}(\mu)-m_{1}^{2}(\mu)}}\right]$, where $\rho$ is some $\boxplus$-infinitely divisible probability measure.

In one's turn Theorem 2.5 is a simple consequence of the following theorem.

Theorem 2.6. Let $\mu \in \mathcal{M}_{d}, d \in \mathbb{N}_{1}$, and there exists a sequence $\left\{\rho_{n}\right\}_{n=1}^{\infty}$ of probability measures such that

$$
\liminf _{n \rightarrow \infty} \frac{\log \Delta\left(\mu^{n \boxplus}, \rho_{n}^{n \boxplus}\right)}{\sqrt{n}}=-\beta<0 .
$$

Then there exists a subsequence of positive integers $\left\{n^{\prime}\right\}$ such that $\rho_{n^{\prime}}$ converges weakly to some probability measure $\rho$ and $\varphi(t ; \mu)=\varphi(t ; \rho), t \in\left[-\frac{\beta}{300 \sqrt{m_{2}(\mu)-m_{1}^{2}(\mu)}}, \frac{\beta}{300 \sqrt{m_{2}(\mu)-m_{1}^{2}(\mu)}}\right]$.

Corollary 2.7. Let $\mu$ and $\rho$ belong to $\mathcal{M}_{d}, d \in \mathbb{N}_{1}$, and

$$
\liminf _{n \rightarrow \infty} \frac{\log \Delta\left(\mu^{n \boxplus}, \rho^{n \boxplus}\right)}{\sqrt{n}}=-\beta<0 .
$$


Then $\varphi(t ; \mu)=\varphi(t ; \rho), t \in\left[-\frac{\beta}{300 \sqrt{m_{2}(\mu)-m_{1}^{2}(\mu)}}, \frac{\beta}{300 \sqrt{m_{2}(\mu)-m_{1}^{2}(\mu)}}\right]$.

Consider the class $\mathcal{M}_{\text {det }}$ of probability measures $\mu$ which are uniquely determined by their moment sequence $\left\{m_{k}(\mu)\right\}_{k=0}^{\infty}$, for details see the introduction of 1 . Then the following theorem holds.

Theorem 2.8. Let $\mu \in \mathcal{M}_{\text {det }}$ and $\mu \notin \mathbf{D}^{\boxplus}$. Then

$$
\liminf _{n \rightarrow \infty} \frac{\log \Delta\left(\mu^{n \boxplus}, \mathbf{D}^{\boxplus}\right)}{\sqrt{n}}=0 .
$$

Since the class $\mathcal{M}_{C}$ of probability measures $\mu$ such that Carleman's condition

$$
\sum_{k=1}^{\infty} \frac{1}{\sqrt[2 k]{m_{2 k}(\mu)}}=\infty
$$

holds is contained in $\mathcal{M}_{\text {det }}$ we note that relation(2.6) holds for $\mu \in \mathcal{M}_{C}$.

The next Theorem 2.9 shows that Theorem 2.4 gives a lower bound which is close to the optimal one. Before to formulate Theorem 2.9 we remark that in the sequel we use slowly varying positive functions $L(x) \geq 1$ with continuous derivative, satisfying the following property: $L(x) \sim L(x / L(x))$ as $x \rightarrow \infty$.

Theorem 2.9. There exists a symmetric probability measure $\mu \notin \mathbf{D}^{\boxplus}$ such that

$$
\Delta\left(\mu^{n \boxplus}, \mathbf{D}^{\boxplus}\right) \leq e^{-c_{4}(\mu) \sqrt{n} / L(n)}, \quad n \in \mathbb{N},
$$

where $L(n) \geq 1$ is a slowly varying positive function such that

$$
\sum_{n=1}^{\infty} \frac{1}{n L(n)}<\infty
$$

Let $\mu \in \mathcal{M}_{2 k}$ with some positive integer $k \geq 1$. Denote by $\alpha_{s}(\mu), s=1, \ldots, 2 k$, free cumulants of of the probability measure $\mu \in \mathcal{M}_{2 k}$ (see the definition of free cumulants in Section 3).

Theorem 2.10. Let $\mu \in \mathcal{M}_{2 k}, k \in \mathbb{N}_{1}$, with $\alpha_{2 k}(\mu)<0$. Assume that there exists $a \boxplus$ infinitely divisible probability measure $\rho \in \mathcal{M}_{2 k}$ such that $\alpha_{s}(\mu)=\alpha_{s}(\rho), s=1, \ldots, 2 k-1$. Then there exist positive constants $c_{5}(\mu, \rho), c_{6}(\mu, \rho)$ depending on $\mu$ and $\rho$ only such that

$$
\frac{c_{5}(\mu, \rho)}{n^{k+2+3 /(k-1)}} \leq \Delta\left(\mu^{n \boxplus}, \rho^{n \boxplus}\right) \leq \min \left(1, \frac{c_{6}(\mu, \rho)}{n^{(k-4) / 4}}\right), \quad n \in \mathbb{N} .
$$

\section{Auxiliary Results}

We need results about some classes of analytic functions (see [1], Section 3).

The class $\mathcal{N}$ (Nevanlinna, R.) is the class of analytic functions $f(z): \mathbb{C}^{+} \rightarrow\{z: \Im z \geq$ $0\}$. For such functions there is the integral representation, for $z \in \mathbb{C}^{+}$,

$$
f(z)=a+b z+\int_{\mathbb{R}} \frac{1+u z}{u-z} \tau(d u)=a+b z+\int_{\mathbb{R}}\left(\frac{1}{u-z}-\frac{u}{1+u^{2}}\right)\left(1+u^{2}\right) \tau(d u),
$$


where $b \geq 0, a \in \mathbb{R}$, and $\tau$ is a non negative finite measure. Moreover, $a=\Re f(i)$ and $\tau(\mathbb{R})=\Im f(i)-b$. From this formula it follows that

$$
f(z)=(b+o(1)) z
$$

for $z \in \mathbb{C}^{+}$such that $|\Re z| / \Im z$ stays bounded as $|z|$ tends to infinity (in other words $z \rightarrow \infty$ non-tangentially to $\mathbb{R}$ ). Hence if $b \neq 0$, then $f$ has a right inverse $f^{(-1)}$ defined on the region

$$
\Gamma_{\alpha, \beta}:=\left\{z \in \mathbb{C}^{+}:|\Re z|<\alpha \Im z, \Im z>\beta\right\}
$$

for any $\alpha>0$ and some positive $\beta=\beta(f, \alpha)$.

A function $f \in \mathcal{N}$ admits the representation

$$
f(z)=\int_{\mathbb{R}} \frac{\sigma(d u)}{u-z}, \quad z \in \mathbb{C}^{+},
$$

where $\sigma$ is a finite non negative measure, if and only if $\sup _{y \geq 1}|y f(i y)|<\infty$.

The Stieltjes-Perron inversion formula for the functions $f$ of class $\mathcal{N}$ has the following form. Let $\psi(u):=\int_{0}^{u}\left(1+t^{2}\right) \tau(d t)$. Then

$$
\psi\left(u_{2}\right)-\psi\left(u_{1}\right)=\lim _{\eta \rightarrow 0} \frac{1}{\pi} \int_{u_{1}}^{u_{2}} \Im f(\xi+i \eta) d \xi,
$$

where $u_{1}<u_{2}$ denote two continuity points of the function $\psi(u)$.

For $\mu \in \mathcal{M}$, consider its Cauchy transform $G_{\mu}(z)$ (see (2.2)). Following Maassen [22] and Bercovici and Voiculescu [7], we shall consider in the following the reciprocal Cauchy transform

$$
F_{\mu}(z)=\frac{1}{G_{\mu}(z)}
$$

The corresponding class of reciprocal Cauchy transforms of all $\mu \in \mathcal{M}$ will be denoted by $\mathcal{F}$. This class coincides with the subclass of Nevanlinna functions $f$ for which $f(z) / z \rightarrow 1$ as $z \rightarrow \infty$ non-tangentially to $\mathbb{R}$. Indeed, reciprocal Cauchy transforms of probability measures have obviously such property. Let $f \in \mathcal{N}$ and $f(z) / z \rightarrow 1$ as $z \rightarrow \infty$ nontangentially to $\mathbb{R}$. Then, by (3.2), $f$ admits the representation (3.1) with $b=1$. By (3.2) and (3.3),$-1 / f(z)$ admits the representation (3.3) with $\sigma \in \mathcal{M}$.

The functions $f$ of the class $\mathcal{F}$ satisfy the inequality

$$
\Im f(z) \geq \Im z, \quad z \in \mathbb{C}^{+} .
$$

The function $\phi_{\mu}(z)=F_{\mu}^{(-1)}(z)-z$ is called the Voiculescu transform of $\mu$ and $\phi_{\mu}(z)$ is an analytic function on $\Gamma_{\alpha, \beta}$ with the property $\Im \phi_{\mu}(z) \leq 0$ for $z \in \Gamma_{\alpha, \beta}$, where $\phi_{\mu}(z)$ is defined. On the domain $\Gamma_{\alpha, \beta}$, where the functions $\phi_{\mu_{1}}(z), \phi_{\mu_{2}}(z)$, and $\phi_{\mu_{1} \boxplus \mu_{2}}(z)$ are defined, we have

$$
\phi_{\mu_{1} \boxplus \mu_{2}}(z)=\phi_{\mu_{1}}(z)+\phi_{\mu_{2}}(z) .
$$


This relation for the distribution $\mu_{1} \boxplus \mu_{2}$ of $X+Y$, where $X$ and $Y$ are free random variables, is due to Voiculescu [28] for the case of compactly supported measures. The result was extended by Maassen [22] to measures with finite variance; the general case was proved by Bercovici and Voiculescu [7].

Assume that $\beta_{k}(\mu)<\infty$ for some $k \in \mathbb{N}$. Then

$$
G_{\mu}(z)=\frac{1}{z}+\frac{m_{1}(\mu)}{z^{2}}+\cdots+\frac{m_{k}(\mu)}{z^{k+1}}+o\left(\frac{1}{z^{k+1}}\right), \quad z \rightarrow \infty, z \in \Gamma_{\alpha, 1} .
$$

It follows from this relation (see for example [18]) that

$$
\phi_{\mu}(z)=\alpha_{1}(\mu)+\frac{\alpha_{2}(\mu)}{z}+\cdots+\frac{\alpha_{k}(\mu)}{z^{k-1}}+o\left(\frac{1}{z^{k-1}}\right), \quad z \rightarrow \infty, z \in \Gamma_{\alpha, 1} .
$$

We call the coefficients $\alpha_{m}(\mu), m=1, \ldots, k$, the free cumulants of the probability measure $\mu$. It is easy to see that $\alpha_{1}(\mu)=m_{1}(\mu), \alpha_{2}(\mu)=m_{2}(\mu)-m_{1}^{2}(\mu), \alpha_{3}(\mu)=m_{3}(\mu)-$ $3 m_{1}(\mu) m_{2}(\mu)+2 m_{1}^{3}(\mu)$. In the case $m_{1}(\mu)=0$ and $m_{2}(\mu)=1$ we have $\alpha_{1}(\mu)=0, \alpha_{2}(\mu)=$ $1, \alpha_{3}(\mu)=m_{3}(\mu)$ and $\alpha_{4}(\mu)=m_{4}(\mu)-2$.

If $\mu \in \mathcal{M}$ has moments of any order, that is $\beta_{k}(\mu)<\infty$ for any $k \in \mathbb{N}$, then there exist cumulants $\alpha_{m}(\mu), m=1, \ldots$, and we can consider the formal power series

$$
\phi_{\mu}(z)=\sum_{m=1}^{\infty} \frac{\alpha_{m}(\mu)}{z^{m-1}} .
$$

In addition $\phi_{\mu}(z)$ satisfies (3.9) for any fixed $k \in \mathbb{N}$. If $\mu$ has a bounded support, $\phi_{\mu}(z)$ is an analytic function on the domain $|z|>R$ with some $R>0$ and the series (3.10) converges absolutely and uniformly for such $z$.

In order to express moments in terms of free cumulants introduce some notations. A partition $\mathbf{V}=\left\{V_{1}, V_{2}, \ldots, V_{s}\right\}$ of the set $[n]:=\{1,2, \ldots, n\}$ consists of nonempty, pairwise disjoint blocks $V_{1}, V_{2}, \ldots, V_{s}$ satisfying $\cup_{j=1}^{s} V_{j}=[n] .\left|V_{j}\right|$ denotes the number of elements of $V_{j}$. $\mathbf{V}$ is called a non-crossing partition if for $V_{j}=\left\{v_{1}, v_{2}, \ldots, v_{p}\right\}$ and $V_{l}=\left\{w_{1}, w_{2}, \ldots, w_{q}\right\}$ we have $w_{m}<v_{1}<w_{m+1}$ if and only if $w_{m}<v_{p}<w_{m+1}(m=$ $1,2, \ldots, q-1)$. Denote by $Q_{n}^{(s)}$ the number of non-crossing partitions of $[n]$ into $s$ blocks. It is well-known, see [17], that

$$
Q_{n}^{(s)}=\frac{1}{n}\left(\begin{array}{c}
n \\
s
\end{array}\right)\left(\begin{array}{c}
n \\
s-1
\end{array}\right) .
$$

The following formula holds

$$
m_{n}(\mu)=\sum_{\mathbf{V} \in N C(n)} \prod_{j=1}^{s} \alpha_{\left|V_{j}\right|}(\mu),
$$

where the summation here is over the non-crossing partitions $\left\{V_{1}, V_{2}, \ldots, V_{s}\right\}$.

Voiculescu [30] showed for compactly supported probability measures that there exist unique functions $Z_{1}, Z_{2} \in \mathcal{F}$ such that $G_{\mu_{1} \boxplus \mu_{2}}(z)=G_{\mu_{1}}\left(Z_{1}(z)\right)=G_{\mu_{2}}\left(Z_{2}(z)\right)$ for all 
$z \in \mathbb{C}^{+}$. Using Speicher's combinatorial approach [26] to freeness, Biane [9] proved this result in the general case.

Bercovici and Belinschi [5], Belinschi [6], Chistyakov and Götze [11 proved, using complex analytic methods, that there exist unique functions $Z_{1}(z)$ and $Z_{2}(z)$ in the class $\mathcal{F}$ such that, for $z \in \mathbb{C}^{+}$,

$$
z=Z_{1}(z)+Z_{2}(z)-F_{\mu_{1}}\left(Z_{1}(z)\right) \quad \text { and } \quad F_{\mu_{1}}\left(Z_{1}(z)\right)=F_{\mu_{2}}\left(Z_{2}(z)\right) .
$$

The function $F_{\mu_{1}}\left(Z_{1}(z)\right)$ belongs again to the class $\mathcal{F}$ and there exists a probability measure $\mu$ such that $F_{\mu_{1}}\left(Z_{1}(z)\right)=F_{\mu}(z)$, where $F_{\mu}(z)=1 / G_{\mu}(z)$ and $G_{\mu}(z)$ is the Cauchy transform as in (2.2).

Specializing to $\mu_{1}=\mu_{2}=\cdots=\mu_{n}=\mu$ write $\mu_{1} \boxplus \ldots \mu_{n}=\mu^{n \boxplus}$. The relation (3.13) admits the following consequence (see for example [11]).

Proposition 3.1. Let $\mu \in \mathcal{M}$. There exists a unique function $Z_{n} \in \mathcal{F}$ such that

$$
z=n Z_{n}(z)-(n-1) F_{\mu}\left(Z_{n}(z)\right), \quad z \in \mathbb{C}^{+},
$$

and $F_{\mu^{n \boxplus}}(z)=F_{\mu}\left(Z_{n}(z)\right)$.

Now we formulate and prove an important auxiliary result about the behaviour of the function $Z_{n}(z)$ from the last proposition.

We obtain from (3.14) the formula

$$
Z_{n}^{(-1)}(z)=n z-(n-1) F_{\mu}(z)
$$

for $z \in \Gamma_{\alpha, \beta}$ with some $\alpha, \beta>0$. By this formula we continue the function $Z_{n}^{(-1)}(z)$ as an analytic function to $\mathbb{C}^{+}$. By (3.1), we have the following representation for the function $F_{\mu}(z)$

$$
F_{\mu}(z)=c+z+\int_{\mathbb{R}} \frac{1+u z}{u-z} \sigma(d u), \quad z \in \mathbb{C}^{+},
$$

where $c \in \mathbb{R}$, and $\sigma$ is a non negative finite measure. Moreover, $c=\Re F_{\mu}(i)$ and $\sigma(\mathbb{R})=$ $\Im F_{\mu}(i)-1$.

Bercovici and Voiculescu [7]) proved the next result.

Proposition 3.2. A probability measure $\mu$ is $\boxplus$-infinitely divisible if and only if the function $\phi_{\mu}(z)$ has an analytic continuation to $\mathbb{C}^{+}$, with values in $\mathbb{C}^{-} \cup \mathbb{R}$, such that

$$
\lim _{y \rightarrow+\infty} \frac{\phi_{\mu}(i y)}{y}=0 .
$$

It follows from Proposition 3.2 and (3.13), (3.16) that a probability measure $\nu_{n}$ such that $F_{\nu_{n}}(z)=Z_{n}(z), z \in \mathbb{C}^{+}$, is $\boxplus$-infinitely divisible.

Consider the functional equation, for every real fixed $x$,

$$
y\left(1-(n-1) \int_{\mathbb{R}} \frac{\left(1+u^{2}\right) \sigma(d u)}{(u-x)^{2}+y^{2}}\right)=0, \quad y>0 .
$$


It is clear that this equation has at most one positive solution. If such solution exists, denote it by $y_{n}(x)>0$ and assume that $y_{n}(x)=0$ otherwise. We note that the curve $\gamma_{n}=\left\{y=y_{n}(x), x \in \mathbb{R}\right\}$ is Jordan one.

Consider the open domain $D_{n}:=\left\{z=x+i y, x, y \in \mathbb{R}: y>y_{n}(x)\right\}$.

Lemma 3.3. The map $Z_{n}(z): \mathbb{C}^{+} \mapsto D_{n}$ is univalent. Moreover the function $Z_{n}(z), z \in$ $\mathbb{C}^{+}$, is continuous up to the real axis and the real axis is mapped onto the curve $\gamma_{n}$.

Lemma 3.4. Let $c_{1}(\mu)>0$ and let $Z_{n}(z)$ be the solution of the equation (3.14). Then the following lower bound holds

$$
\left|Z_{n}(z)\right| \geq \frac{1}{4} \sqrt{c_{1}(\mu)(n-1)}, \quad z \in \mathbb{C}^{+}, \quad n \geq c(\mu) .
$$

The lemmas 3.3 and 3.4 were proved in [14].

We need the following Bercovici-Voiculescu result [7].

Proposition 3.5. If $\mu, \mu^{\prime}, \nu$, and $\nu^{\prime}$ are probability measures, then

$$
\Delta\left(\mu \boxplus \nu, \mu^{\prime} \boxplus \nu^{\prime}\right) \leq \Delta\left(\mu, \mu^{\prime}\right)+\Delta\left(\nu, \nu^{\prime}\right) .
$$

In the sequel we use the following well-known inequality (see [21]).

Proposition 3.6. Let $Q(x), x \in \mathbb{R}$ be a distribution function. Then

$$
\int_{\{|x|>2 / u\}} d Q(x) \leq \frac{2}{u} \int_{[0, u]}(1-\Re \varphi(t ; Q)) d t, \quad u>0 .
$$

Lemma 3.7. If a probability measure $\mu$ has a finite second moment $m_{2}(\mu)$, then the solution of the equation (3.14) $Z_{n}(z)$ admits the representation

$$
Z_{n}(z)=z-(n-1) m_{1}(\mu)+\int_{\mathbb{R}} \frac{\tau(d u)}{u-z}, \quad z \in \mathbb{C}^{+},
$$

whith a non negative measure $\tau$ such that $\tau(\mathbb{R})=(n-1)\left(m_{2}(\mu)-m_{1}^{2}(\mu)\right)$.

Proof. Since $F_{\mu}(z):=1 / G_{\mu}(z) \in \mathcal{N}$ and $F_{\mu}(i y) /(i y) \rightarrow 1$ as $y \rightarrow \infty$, we have from Nevanlinna's integral representation $F_{\mu}(z)=z-m_{1}(\mu)+f(z)$, where $f \in \mathcal{N}$ and $f(i y) / y \rightarrow 0$ as $y \rightarrow \infty$. In view of the relation

$$
\lim _{y \rightarrow \infty}(i y)^{3}\left(G_{\mu}(i y)-\frac{1}{i y}-\frac{m_{1}(\mu)}{(i y)^{2}}\right)=m_{2}(\mu),
$$

we have, for $y \rightarrow \infty$,

$$
F_{\mu}(i y)-i y=-m_{1}(\mu)-\frac{m_{2}(\mu)-m_{1}^{2}(\mu)}{i y}+o\left(\frac{1}{y}\right) .
$$

From here we conclude that

$$
f(z)=\int_{\mathbb{R}} \frac{\sigma(d u)}{u-z}
$$


whith the measure $\sigma$ such that $\sigma(\mathbb{R})=m_{2}(\mu)-m_{1}^{2}(\mu)$.

The solution $Z_{n}(z)$ of (3.14) belongs to the class $\mathcal{F}$, therefore $Z_{n}(z)=z+g(z), z \in \mathbb{C}^{+}$, where $g \in \mathcal{N}$ and $g(i y) / y \rightarrow 0$ as $y \rightarrow \infty$. Rewrite (3.14) in the form

$$
\frac{1}{n-1}\left(Z_{n}(z)-z\right)=-m_{1}(\mu)+\int_{\mathbb{R}} \frac{\sigma(d u)}{u-Z_{n}(z)} .
$$

Since $\sup _{y \geq 1}|y f(i y)|<\infty$, we see that

$$
Z_{n}(z)-z=-(n-1) m_{1}(\mu)+\int_{\mathbb{R}} \frac{\tau(d u)}{u-z}, \quad z \in \mathbb{C}^{+},
$$

where $\tau$ is a finite non negative measure. In addition, by the relations $-\lim _{y \rightarrow \infty}$ iy $f($ iy $)$ $\sigma(\mathbb{R})=m_{2}(\mu)-m_{1}^{2}(\mu)$ and $\lim _{y \rightarrow \infty}(i y)\left(Z_{n}(i y)-i y+(n-1) m_{1}(\mu)\right)=\tau(\mathbb{R})$, we conclude that $\tau(\mathbb{R})=(n-1)\left(m_{2}(\mu)-m_{1}^{2}(\mu)\right)$. The lemma is proved.

The next lemma is a refinement of Lemma 3.7. We save all notation of this lemma.

Lemma 3.8. Let a probability measure $\mu$ has compact support, i.e. $\mu(\mathbb{R})=\mu([-R, R])$ with some $R>0$. Then the solution of (3.14) $Z_{n}(z)$ admits the representation (3.19), where $\tau(\mathbb{R})=\tau\left(\left[-R_{1}, R_{1}\right]\right)$ with $R_{1}=4(n-1)(R+1)(\sigma(\mathbb{R})+1)$.

Proof. The function $F_{\mu}(z)$ is analytic in $\mathbb{C} \backslash[-R, R]$ and is real-valued for $\mathbb{R} \backslash[-R, R]$. Therefore $\sigma$ from (3.16) has compact support and $\sigma(\mathbb{R})=\sigma([-R, R])$.

Denote by $\mu_{Z_{n}}$ the probability measure with the Cauchy transform $\frac{1}{Z_{n}(z)}$. By (3.21), we see that

$$
\alpha_{1}\left(\mu_{Z_{n}}\right)=(n-1) m_{1}(\mu), \quad \alpha_{k}\left(\mu_{Z_{n}}\right)=(n-1) s_{k-1}(\sigma), \quad k=2,3, \ldots,
$$

where $s_{k-1}(\sigma)=\int_{\mathbb{R}} u^{k-1} \sigma(d u)$. Therefore we have the bounds

$$
\left|\alpha_{k}\left(\mu_{Z_{n}}\right)\right| \leq(n-1) R^{k-1} \sigma(\mathbb{R}) \leq((n-1)(R+1)(\sigma(\mathbb{R})+1))^{k}
$$

for $k=2, \ldots$ Applying these estimates to (3.12), we obtain

$$
\left|m_{k}\left(\mu_{Z_{n}}\right)\right| \leq(4(n-1)(R+1)(\sigma(\mathbb{R})+1))^{k}
$$

for $k=1,2, \ldots$ See [23], p.p. 218-219, as well.

The function $G_{\mu_{Z_{n}}}(z)$ is analytic in the domain $|z=x+i y|>R_{1}$, is not equal to zero and real-valued for $|x|>R_{1}$. Therefore $Z_{n}(z)$ is analytic for $|z|>R_{1}$ and real-valued for $|x|>R_{1}$ as well. Therefore we obtain the assertion of the lemma from (3.22) and from the inversion formula (3.4). 


\section{Approximations in Limit Theorems}

Proof of Theorem 2.6. In the sequel we assume without loss of generality $m_{1}(\mu)=0$

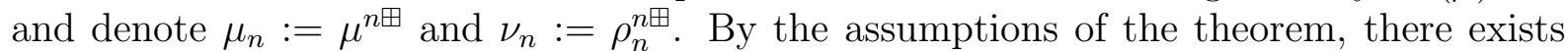
a subsequence of increasing positive integers $\left\{n_{k}\right\}_{k=1}^{\infty}$ such that

$$
\Delta\left(\mu_{n_{k}}, \nu_{n_{k}}\right) \leq \varepsilon_{n_{k}}:=e^{-\beta^{\prime} \sqrt{n_{k}}}
$$

where $0<\beta^{\prime}<\beta$. By the assumptions of the theorem, $\beta_{d}(\mu)<\infty, d \geq 3$, therefore, in view of the well-known Minkowski inequality $\left(\beta_{d}\left(\mu^{n \boxplus}\right)\right)^{1 / d} \leq n\left(\beta_{d}(\mu)\right)^{1 / d}$ (see [27]), we have

$$
\mu_{n}(\mathbb{R} \backslash[-t, t]) \leq n^{d} \beta_{d}(\mu) t^{-d} \text { for all } t>0 .
$$

By (3.8), $G_{\mu}(z)=\frac{1}{z}+\frac{m_{2}(\mu)}{z^{3}}+o\left(\frac{1}{z^{3}}\right)$, when $z \rightarrow \infty$ in the angle $\delta \leq \arg z \leq \pi-\delta$ for any small fixed $\delta>0$. Therefore $F_{\mu}(z)=1 / G_{\mu}(z)=z-\frac{m_{2}(\mu)}{z}+o\left(\frac{1}{z}\right)$ for the same $z$. Hence, by (3.3),

$$
F_{\mu}(z)=z+\int_{\mathbb{R}} \frac{\sigma(d u)}{u-z}, \quad z \in \mathbb{C}^{+},
$$

where $\sigma$ is a non negative finite measure such that $\sigma(\mathbb{R})=m_{2}(\mu)$.

By Proposition 3.1, there exists a unique function $Z_{n}(z) \in \mathcal{F}$ such that

$$
z=n Z_{n}(z)-(n-1) F_{\mu}\left(Z_{n}(z)\right), \quad z \in \mathbb{C}^{+},
$$

and $G_{\mu_{n}}(z)=G_{\mu}\left(Z_{n}(z)\right), z \in \mathbb{C}^{+}$. By Lemma 3.7, we conclude that $Z_{n}(z)$ admits the representation

$$
Z_{n}(z)=z+\int_{\mathbb{R}} \frac{\sigma_{n}(d u)}{u-z}, \quad z \in \mathbb{C}^{+},
$$

where $\sigma_{n}$ is a non negative finite measure such that $\sigma_{n}(\mathbb{R})=(n-1) m_{2}(\mu)$.

By Proposition 3.1, there exists a unique function $W_{n}(z) \in \mathcal{F}$ such that

$$
z=n W_{n}(z)-(n-1) F_{\rho_{n}}\left(W_{n}(z)\right), \quad z \in \mathbb{C}^{+},
$$

and $G_{\nu_{n}}(z)=G_{\rho_{n}}\left(W_{n}(z)\right), z \in \mathbb{C}^{+}$.

Now we evaluate the closeness of the functions $Z_{n}(z)$ and $W_{n}(z)$.

Lemma 4.1. The following upper bound holds

$$
\left|Z_{n}(z)-W_{n}(z)\right| \leq \frac{18 \pi \varepsilon_{n}}{\Im z}\left(|z|+\frac{n m_{2}(\mu)}{\Im z}\right)^{2}
$$

for $|\Re z| \leq R_{n}$ and $\Im z \geq 1 / R_{n}$, where $R_{n}:=\left(10 \sqrt{\varepsilon_{n} n m_{2}(\mu)}\right)^{-1}$.

Proof. Integrating by parts we obtain the formula

$$
r_{n}(z):=G_{\mu_{n}}(z)-G_{\nu_{n}}(z)=-\int_{\mathbb{R}}\left(\mu_{n}((-\infty, u))-\nu_{n}((-\infty, u))\right) \frac{d u}{(z-u)^{2}}, \quad z \in \mathbb{C}^{+} .
$$


Using (4.1), we easily deduce from this formula the estimate

$$
\left|r_{n}(z)\right| \leq \varepsilon_{n} \int_{\mathbb{R}} \frac{d u}{|z-u|^{2}}=\frac{\pi \varepsilon_{n}}{\Im z}, \quad z \in \mathbb{C}^{+} .
$$

By (4.4) and (4.6), we have

$$
r_{n}(z)=n(n-1) \frac{W_{n}(z)-Z_{n}(z)}{\left(n Z_{n}(z)-z\right)\left(n W_{n}(z)-z\right)}
$$

and hence

$$
W_{n}(z)-Z_{n}(z)=\frac{r_{n}(z)}{n(n-1)} \frac{\left(n Z_{n}(z)-z\right)^{2}}{1-r_{n}(z)\left(n Z_{n}(z)-z\right) /(n-1)} .
$$

By (4.5), we see that

$$
\left|Z_{n}(z)\right| \leq|z|+(n-1) m_{2}(\mu) \frac{1}{\Im z}, \quad z \in \mathbb{C}^{+} .
$$

By (4.8) and (4.10), we conclude that

$$
\frac{\left|r_{n}(z)\right|}{n-1}\left(n\left|Z_{n}(z)\right|+|z|\right) \leq \frac{\pi n m_{2}(\mu) \varepsilon_{n}}{(\Im z)^{2}}+\frac{3 \pi \varepsilon_{n}|z|}{\Im z}<\frac{1}{5}
$$

for $|\Re z| \leq R_{n}$ and $\Im z \geq 1 / R_{n}$. Using (4.10) and (4.11) we deduce from (4.9)

$$
\begin{aligned}
& \left|W_{n}(z)-Z_{n}(z)\right| \leq \frac{\left|r_{n}(z)\right|}{n(n-1)} \frac{\left(n\left|Z_{n}(z)\right|+|z|\right)^{2}}{|1-| r_{n}(z)\left|\left(n\left|Z_{n}(z)\right|+|z|\right) /(n-1)\right|} \\
& \leq 2 \frac{\pi \varepsilon_{n}}{\Im z}\left(3|z|+\frac{n m_{2}(\mu)}{\Im z}\right)^{2}
\end{aligned}
$$

for $|\Re z| \leq R_{n}$ and $\Im z \geq 1 / R_{n}$. The lemma is proved.

In the sequel we need an information about Nevalinna functions $\frac{1}{u-Z_{n}(z)}, z \in \mathbb{C}^{+}$for all $u \in \mathbb{R}$.

Lemma 4.2. For every $u \in \mathbb{R}$, Nevalinna functions $\frac{1}{u-Z_{n}(z)}, z \in \mathbb{C}^{+}$, admit the integral representation

$$
\frac{1}{u-Z_{n}(z)}=\int_{\mathbb{R}} \frac{\zeta_{n}(u ; d s)}{s-z},
$$

where $\zeta_{n}(u ; d s)$ are probability measures such that

$$
\zeta_{n}\left(u ;\left[u-\sqrt{2 \sigma_{n}(\mathbb{R})}, u+\sqrt{2 \sigma_{n}(\mathbb{R})}\right]\right) \geq \frac{1}{2}
$$

for all $u \in \mathbb{R}$ and $n \in \mathbb{N}$. 
Proof. By (4.3), we have the relation $Z_{n}(z)=z-\sigma_{n}(\mathbb{R}) / z+o(1 / z)$ as $z \rightarrow \infty$ and $z \in \Gamma_{\alpha, \beta}$ for some positive $\alpha$ and $\beta$. Using it we easily deduce, for $z \rightarrow \infty$ and $z \in \Gamma_{\alpha, \beta}$,

$$
\frac{1}{u-Z_{n}(z)}=-\frac{1}{z}-\frac{u}{z^{2}}-\frac{\sigma_{n}(\mathbb{R})+u^{2}}{z^{3}}+\frac{o(1)}{z^{3}} .
$$

By Lemma 3.8, we conclude that $m_{1}\left(\zeta_{n}(u ; d s)\right)=u$ and $m_{2}\left(\zeta_{n}(u ; d s)\right)=u^{2}+\sigma_{n}(\mathbb{R})$. Then, by Chebyshev's inequality, we have the bound

$$
\zeta_{n}\left(u ; \mathbb{R} \backslash\left[u-\sqrt{2 \sigma_{n}(\mathbb{R})}, u+\sqrt{2 \sigma_{n}(\mathbb{R})}\right]\right) \leq \frac{\sigma_{n}(\mathbb{R})}{2 \sigma_{n}(\mathbb{R})}=\frac{1}{2} .
$$

The lemma is proved.

Our next step is to prove that the tails of the measure $\rho_{n}$ are small. Denote

$$
\varepsilon_{n}^{*}:=\varepsilon_{n}+\left(\frac{n}{N_{n}}\right)^{3}+\frac{\eta_{n}}{N_{n}}+\varepsilon_{n}\left(\frac{N_{n}}{\eta_{n}^{3}}+\frac{n^{2}}{\eta_{n}^{5}}\right),
$$

where $N_{n}=\varepsilon_{n}^{-1 / 13}$ and $\eta_{n}=1 / N_{n}^{2}$.

Lemma 4.3. The following upper bounds hold

$$
\rho_{n}\left(\left(-\infty,-2 N_{n}\right)\right)+\rho_{n}\left(\left(2 N_{n}, \infty\right)\right) \leq c(\mu) \varepsilon_{n}^{*} .
$$

Proof. Using (4.1) and (4.2), we get the inequality

$$
\begin{aligned}
& -\int_{\left[N_{n}, \infty\right)} \Im G_{\nu_{n}}\left(x+i \eta_{n}\right) d x=\int_{\mathbb{R}} \nu_{n}(d s) \int_{\left[N_{n}, \infty\right)} \frac{\eta_{n} d x}{(x-s)^{2}+\eta_{n}^{2}} \\
& \left.=\int_{\left[-N_{n} / 2, N_{n} / 2\right]}+\int_{\mathbb{R} \backslash\left[-N_{n} / 2, N_{n} / 2\right]}\right) \nu_{n}(d s) \int_{\left[\left(N_{n}-s\right) / \eta_{n}, \infty\right)} \frac{d t}{t^{2}+1} \\
& \leq \pi \nu_{n}\left(\mathbb{R} \backslash\left[-N_{n} / 2, N_{n} / 2\right]\right)+\frac{2 \eta_{n}}{N_{n}} \leq 2 \pi \varepsilon_{n}+\frac{8 \pi \beta_{3}(\mu) n^{3}}{N_{n}^{3}}+\frac{2 \eta_{n}}{N_{n}} .
\end{aligned}
$$

On the other hand we have the relation

$$
\begin{aligned}
-\int_{\left[N_{n}, \infty\right)} \Im G_{\nu_{n}}\left(x+i \eta_{n}\right) d x & =\int_{\left[N_{n}, \infty\right)} d x \int_{\mathbb{R}} \Im \frac{1}{u-W_{n}\left(x+i \eta_{n}\right)} \rho_{n}(d u) \\
& =\int_{\mathbb{R}} \rho_{n}(d u) \int_{\left[N_{n}, \infty\right)} \Im \frac{1}{u-W_{n}\left(x+i \eta_{n}\right)} d x .
\end{aligned}
$$

In view of Lemma 4.1, we conclude

$$
\begin{aligned}
\left|\Im\left(\frac{1}{u-Z_{n}(z)}-\frac{1}{u-W_{n}(z)}\right)\right| & =\left|\Im \frac{W_{n}(z)-Z_{n}(z)}{\left(u-Z_{n}(z)\right)\left(u-W_{n}(z)\right)}\right| \leq \frac{\left|W_{n}(z)-Z_{n}(z)\right|}{(\Im z)^{2}} \\
& \leq \frac{18 \pi \varepsilon_{n}}{(\Im z)^{3}}\left(|z|+\frac{n m_{2}(\mu)}{\Im z}\right)^{2}
\end{aligned}
$$


for $|\Re z| \leq R_{n}$ and $\Im z \geq 1 / R_{n}$. Note that $R_{n} \geq N_{n}$ for sufficiently large $n$. Applying (4.16) to (4.15) and recalling (4.12), we obtain from (4.14) the lower bound

$$
c(\mu) \varepsilon_{n}^{*} \geq c(\mu) \varepsilon_{n}\left(\frac{N_{n}}{\eta_{n}^{3}}+\frac{n^{2}}{\eta_{n}^{5}}\right) \geq \int_{\mathbb{R}} \rho_{n}(d u) \int_{\left[N_{n}, \infty\right.} \Im \frac{1}{u-Z_{n}\left(x+i \eta_{n}\right)} d x .
$$

By Lemma 4.2, we deduce the inequality, for $u \geq 2 N_{n}$,

$$
\begin{aligned}
& \int_{\left[N_{n}, \infty\right)} \Im \frac{1}{u-Z_{n}\left(x+i \eta_{n}\right)} d x=\int_{\left[N_{n}, \infty\right)} \int_{\mathbb{R}} \frac{\eta_{n} \zeta(u ; d s)}{(s-x)^{2}+\eta_{n}^{2}} d x \\
& =\int_{\mathbb{R}} \int_{\left(N_{n}-s\right) / \eta_{n}}^{\infty} \frac{d v}{v^{2}+1} \zeta(u ; d s) \geq \int_{\left[u-\sqrt{2 \sigma_{n}(\mathbb{R})}, u+\sqrt{2 \sigma_{n}(\mathbb{R})}\right]} \int_{-N_{n} /\left(2 \eta_{n}\right)}^{N_{n} /\left(2 \eta_{n}\right)} \frac{d v}{v^{2}+1} \zeta(u ; d s) \\
& \geq \frac{\pi}{2} \int_{\left[u-\sqrt{2 \sigma_{n}(\mathbb{R})}, u+\sqrt{\left.2 \sigma_{n}(\mathbb{R})\right]}\right.} \zeta(u ; d s) \geq \frac{\pi}{4} .
\end{aligned}
$$

From (4.17) and (4.18) it follows that $\int_{\left[2 N_{n}, \infty\right)} \rho_{n}(d u) \leq c(\mu) \varepsilon_{n}^{*}$. In the same way we obtain the estimate $\int_{\left(-\infty,-2 N_{n}\right]} \rho_{n}(d u) \leq c(\mu) \varepsilon_{n}^{*}$ as well. Thus, the lemma is proved.

Let $\nu \in \mathcal{M}$. In the sequel we denote by $\tilde{\nu}$ a probability measure such that $\tilde{\nu}(S)=\nu(S)$ for all Borel sets $S \subseteq\left[-2 N_{n}, 2 N_{n}\right] \backslash\{0\}$ and $\tilde{\nu}(\{0\})=\nu(\{0\})+\nu\left(\mathbb{R} \backslash\left[-2 N_{n}, 2 N_{n}\right]\right)$.

Now in place of the measures $\mu$ and $\rho_{n}$ we will consider probability measures $\tilde{\mu}$ and $\tilde{\rho}_{n}$. By the inequality $\mu\left(\mathbb{R} \backslash\left[-2 N_{n}, 2 N_{n}\right]\right) \leq \beta_{d}(\mu) /\left(2 N_{n}\right)^{d}$ and Lemma 4.3, $\Delta(\tilde{\mu}, \mu) \leq$ $\beta_{d}(\mu) /\left(2 N_{n}\right)^{d}$ and $\Delta\left(\tilde{\rho}_{n}, \rho_{n}\right) \leq c(\mu) \varepsilon_{n}^{*}$. By (4.1) and Proposition 3.5, we have the following upper bound

$$
\Delta\left(\tilde{\mu}_{n}, \tilde{\nu}_{n}\right) \leq \Delta\left(\tilde{\mu}_{n}, \mu_{n}\right)+\Delta\left(\mu_{n}, \nu_{n}\right)+\Delta\left(\nu_{n}, \tilde{\nu}_{n}\right) \leq c(\mu) n \varepsilon_{n}^{*}
$$

where $\tilde{\mu}_{n}:=\tilde{\mu}^{n \boxplus}$ and $\tilde{\nu}_{n}:=\tilde{\rho}_{n}^{n \boxplus}$. Since $\tilde{\mu}\left(\left[-2 N_{n}, 2 N_{n}\right]\right)=\tilde{\rho}_{n}\left(\left[-2 N_{n}, 2 N_{n}\right]\right)=1$, we conclude that $\tilde{\mu}_{n}\left(\left[-2 n N_{n}, 2 n N_{n}\right]\right)=\tilde{\nu}_{n}\left(\left[-2 n N_{n}, 2 n N_{n}\right]\right)=1$.

The function $F_{\tilde{\mu}}(z)$ admits the representation

$$
F_{\tilde{\mu}}(z)=z+m_{1}(\tilde{\mu})+\int_{\mathbb{R}} \frac{\tilde{\sigma}(d u)}{u-z}, \quad z \in \mathbb{C}^{+},
$$

where $\tilde{\sigma}$ is a non negative finite measure such that $\tilde{\sigma}(\mathbb{R})=m_{2}(\tilde{\mu})-m_{1}^{2}(\tilde{\mu})$. This representation we obtain in the way as we have got representation (4.3).

By Proposition 3.1, there exists a unique function $\tilde{Z}_{n}(z) \in \mathcal{F}$ such that

$$
z=n \tilde{Z}_{n}(z)-(n-1) F_{\tilde{\mu}}\left(\tilde{Z}_{n}(z)\right), \quad z \in \mathbb{C}^{+},
$$


and $G_{\tilde{\mu}_{n}}(z)=G_{\tilde{\mu}}\left(\tilde{Z}_{n}(z)\right), z \in \mathbb{C}^{+}$. Note that, by Lemma 3.7, we conclude that $\tilde{Z}_{n}(z)$ admits the representation

$$
\tilde{Z}_{n}(z)=z-(n-1) m_{1}(\tilde{\mu})+\int_{\mathbb{R}} \frac{\tilde{\sigma}_{n}(d u)}{u-z}, \quad z \in \mathbb{C}^{+}
$$

where $\tilde{\sigma}_{n}$ is a non negative finite measure such that $\tilde{\sigma}_{n}(\mathbb{R})=(n-1)\left(m_{2}(\tilde{\mu})-m_{1}^{2}(\tilde{\mu})\right)$.

In the same way, by Proposition 3.1 there exists a unique function $\tilde{W}_{n}(z) \in \mathcal{F}$ such that

$$
z=n \tilde{W}_{n}(z)-(n-1) F_{\tilde{\rho}_{n}}\left(\tilde{W}_{n}(z)\right), \quad z \in \mathbb{C}^{+},
$$

and $G_{\tilde{\nu}_{n}}(z)=G_{\tilde{\rho}_{n}}\left(\tilde{W}_{n}(z)\right), z \in \mathbb{C}^{+}$. By Lemma 3.7, $\tilde{W}_{n}(z)$ admits the representation

$$
\tilde{W}_{n}(z)=z-(n-1) m_{1}\left(\tilde{\rho}_{n}\right)+\int_{\mathbb{R}} \frac{\left.\tilde{\tau}_{n} d u\right)}{u-z}, \quad z \in \mathbb{C}^{+}
$$

where $\tilde{\tau}_{n}$ is a non-negative finite measure such that $\tilde{\tau}_{n}(\mathbb{R})=(n-1)\left(m_{2}\left(\tilde{\rho}_{n}\right)-m_{1}^{2}\left(\tilde{\rho}_{n}\right)\right)$.

Now we need to estimate closeness of the functions $\tilde{Z}_{n}(z)$ and $\tilde{W}_{n}(z)$.

Lemma 4.4. The following bound holds

$$
\left|\tilde{Z}_{n}(z)-\tilde{W}_{n}(z)\right| \leq c(\mu) n^{3} N_{n}^{2} \varepsilon_{n}^{*} \quad \text { for } \quad 1 \leq \Im z \leq 6 n N_{n} .
$$

Proof. We prove this lemma using the arguments of the proof of Lemma 4.1. Integrating by parts and using (4.19) we obtain the following estimate for $\tilde{r}_{n}(z):=G_{\tilde{\mu}_{n}}(z)-G_{\tilde{\nu}_{n}}(z)$

$$
\left|\tilde{r}_{n}(z)\right| \leq c(\mu) n \varepsilon_{n}^{*} \int_{\left[-2 n N_{n}, 2 n N_{n}\right]} \frac{d u}{|z-u|^{2}} \leq \begin{cases}\frac{c(\mu) n \varepsilon_{n}^{*}}{(\Im z))^{2}}, & \text { if }|\Re z| \leq 6 n N_{n}, \Im z>0 \\ \frac{c(\mu) n^{2} N_{n} \varepsilon_{n}^{*}}{|z|^{2}}, & \text { if }|\Re z|>6 n N_{n}, \Im z>0 .\end{cases}
$$

By (4.21) and (4.23), we have

$$
\tilde{r}_{n}(z)=n(n-1) \frac{\tilde{W}_{n}(z)-\tilde{Z}_{n}(z)}{\left(n \tilde{Z}_{n}(z)-z\right)\left(n \tilde{W}_{n}(z)-z\right)}
$$

and hence

$$
\tilde{W}_{n}(z)-\tilde{Z}_{n}(z)=\frac{\tilde{r}_{n}(z)}{n(n-1)} \frac{\left(n \tilde{Z}_{n}(z)-z\right)^{2}}{1-\tilde{r}_{n}(z)\left(n \tilde{Z}_{n}(z)-z\right) /(n-1)} .
$$

Repeating the arguments which we used in the proof of Lemma 4.1 one can easily obtain the following estimate, for $|\Re z| \leq 6 n N_{n}$, Эz $\geq 1$,

$$
\left|\tilde{Z}_{n}(z)-\tilde{W}_{n}(z)\right| \leq c(\mu) n \varepsilon_{n}^{*}(|z|+n)^{2} .
$$

From (4.22) it follows the upper bound

$$
\left|\tilde{Z}_{n}(z)\right| \leq|z|+(n-1)\left(\left|m_{1}(\tilde{\mu})\right|+m_{2}(\tilde{\mu})\right), \quad \Im z \geq 1 .
$$


By (4.25) and (4.28), we conclude that, for $|\Re z|>6 n N_{n}, \Im z \geq 1$,

$$
\begin{aligned}
\left|\tilde{r}_{n}(z)\right|\left(2\left|\tilde{Z}_{n}(z)\right|+\frac{|z|}{n-1}\right) & \leq c(\mu) \frac{n^{2} N_{n} \varepsilon_{n}^{*}}{|z|^{2}}\left(|z|+(n-1)\left(\left|m_{1}(\tilde{\mu})\right|+m_{2}(\tilde{\mu})\right)\right) \\
& \leq \frac{c(\mu) n^{2} N_{n} \varepsilon_{n}^{*}}{|z|}<\frac{1}{2}, \quad n \geq c(\mu) .
\end{aligned}
$$

Using (4.25), (4.28) and (4.29) we obtain from (4.26) the assertion of the lemma for $1 \leq \Im z \leq 6 n N_{n}$ and $|\Re z|>6 n N_{n}$. Recalling (4.27), we arrive at the assertion of the lemma.

Denote $S_{n}(z):=G_{\tilde{\mu}}\left(\tilde{Z}_{n}(z)\right)-G_{\tilde{\rho}_{n}}\left(\tilde{W}_{n}(z)\right)$ for $z \in \mathbb{C}^{+}$and research the behavior of this function. We represent this function as follows

$$
S_{n}(z)=T_{n}(z)+R_{n}(z):=\int_{\left[-2 N_{n}, 2 N_{n}\right]} \frac{\left(\tilde{\mu}-\tilde{\rho}_{n}\right)(d u)}{\tilde{Z}_{n}(z)-u}+\int_{\left[-2 N_{n}, 2 N_{n}\right]} \frac{\left(\tilde{W}_{n}(z)-\tilde{Z}_{n}(z)\right) \tilde{\rho}_{n}(d u)}{\left(\tilde{Z}_{n}(z)-u\right)\left(\tilde{W}_{n}(z)-u\right)}
$$

for $z \in \mathbb{C}^{+}$.

Here we need to estimate the function $R_{n}(z)$. This is done in the next lemma.

Lemma 4.5. The following upper bound holds

$$
\left|R_{n}(z)\right| \leq c(\mu) n^{3} N_{n}^{2} \varepsilon_{n}^{*} \int_{\left[-2 N_{n}, 2 N_{n}\right]} \frac{\tilde{\rho}_{n}(d u)}{\left|\tilde{Z}_{n}(z)-u\right|^{2}}, \quad 1 \leq \Im z \leq 6 n N_{n} .
$$

Proof. We note, using Lemma 4.4 and (3.6) , that, for $|u| \leq 2 N_{n}$ and $\Im z \geq 1$,

$$
\begin{aligned}
\left|\tilde{W}_{n}(z)-u\right| & \geq\left|\tilde{Z}_{n}(z)-u\right|-\left|\tilde{W}_{n}(z)-\tilde{Z}_{n}(z)\right| \\
& \geq \frac{1}{2}\left|\tilde{Z}_{n}(z)-u\right|+\frac{1}{2} \Im z-c(\mu) n^{3} N_{n}^{2} \varepsilon_{n}^{*} \geq \frac{1}{2}\left|\tilde{Z}_{n}(z)-u\right| .
\end{aligned}
$$

From (4.31) and Lemma 4.4 it follows, for $|u| \leq 2 N_{n}$ and $1 \leq \Im z \leq 6 n N_{n}$,

$$
\left|\frac{\tilde{W}_{n}(z)-\tilde{Z}_{n}(z)}{\left(\tilde{Z}_{n}(z)-u\right)\left(\tilde{W}_{n}(z)-u\right)}\right| \leq \frac{c(\mu) n^{3} N_{n}^{2} \varepsilon_{n}^{*}}{\left|\tilde{Z}_{n}(z)-u\right|^{2}} .
$$

Using this estimate we arrive immediately at the assertion of the lemma.

In order to complete the proof of the theorem we need an additional information about the function $\tilde{Z}_{n}(z)$.

We obtain from (4.21) the following expression for $\tilde{Z}_{n}^{(-1)}(z)$

$$
\tilde{Z}_{n}^{(-1)}(z)=n z-(n-1) F_{\tilde{\mu}}(z)
$$

for $z \in \Gamma_{\alpha, \beta}$ with some $\alpha, \beta>0$. By this formula, we continue the function $\tilde{Z}_{n}^{(-1)}(z)$ as an analytic function in $\mathbb{C}^{+}$. 
Using the representaion (4.20) we consider the functional equation, for every fixed $x \in \mathbb{R}$,

$$
y\left(1-(n-1) \int_{\left[-2 N_{n}, 2 N_{n}\right]} \frac{\tilde{\sigma}(d u)}{(u-x)^{2}+y^{2}}\right)=0, \quad y>0 .
$$

This equation has at most one positive solution. Denote it by $\tilde{y}_{n}(x)>0$ if such a solution exists and by $\tilde{y}_{n}(x)=0$ otherwise. We see that the curve $\tilde{\gamma}_{n}$ given by the equation $y=\tilde{y}_{n}(x), x \in \mathbb{R}$, is a Jordan curve and, as it is easy to see, that $\tilde{y}_{n}(x)=0$ for $|x|>$ $2 N_{n}+\sqrt{\tilde{\sigma}(\mathbb{R})(n-1)}$ and $\tilde{y}_{n}(x)<\sqrt{\tilde{\sigma}(\mathbb{R})(n-1)}$ for $x \in \mathbb{R}$.

Consider the open domain $\tilde{D}_{n}:=\left\{z=x+i y, x, y \in \mathbb{R}: y>\tilde{y}_{n}(x)\right\}$. By Lemma 3.3. the map $\tilde{Z}_{n}(z): \mathbb{C}^{+} \mapsto \tilde{D}_{n}$ is univalent. Moreover the function $\tilde{Z}_{n}(z), z \in \mathbb{C}^{+}$, is continuous up to the real axis and the real axis passages on the curve $\tilde{\gamma}_{n}$.

Let the curve $\gamma_{n}$ be given by the equation $w=\tilde{Z}_{n}^{(-1)}\left(x+i h_{n}\right), x \in \mathbb{R}$, where $h_{n}:=$ $10 \sqrt{\tilde{\sigma}(\mathbb{R}) n}$. By (4.20) and (4.32), we easily get, for all real $x$,

$$
\frac{1}{2} h_{n} \leq \Im \tilde{Z}_{n}^{(-1)}\left(x+i h_{n}\right)=h_{n}\left(1-(n-1) \int_{\left[-2 N_{n}, 2 N_{n}\right]} \frac{\tilde{\sigma}(d u)}{(x-u)^{2}+h_{n}^{2}}\right) \leq h_{n}
$$

and, for $|x| \geq 2 N_{n}+2 h_{n}$

$$
\left|\Re \tilde{Z}_{n}^{(-1)}\left(x+i h_{n}\right)\right|=\left|x-m_{1}(\tilde{\mu})-(n-1) \int_{\left[-2 N_{n}, 2 N_{n}\right]} \frac{(x-u) \tilde{\sigma}(d u)}{(x-u)^{2}+h_{n}^{2}}\right| \geq \frac{1}{2}|x| .
$$

Return to formula (4.30) and write with its help the relation

$$
\int_{\left[-2 n N_{n}, 2 n N_{n}\right]} \frac{\left(\tilde{\mu}_{n}-\tilde{\nu}_{n}\right)(d u)}{\tilde{Z}_{n}^{(-1)}\left(x+i h_{n}\right)-u}=\int_{\left[-2 N_{n}, 2 N_{n}\right]} \frac{\left(\tilde{\mu}-\tilde{\rho}_{n}\right)(d u)}{x-u+i h_{n}}+R_{n}\left(\tilde{Z}_{n}^{(-1)}\left(x+i h_{n}\right)\right)
$$

for $x \in \mathbb{R}$. By Lemma 4.5, we have the estimate

$$
\left|R_{n}\left(\tilde{Z}_{n}^{(-1)}\left(x+i h_{n}\right)\right)\right| \leq c(\mu) n^{3} N_{n}^{2} \varepsilon_{n}^{*} \int_{\left[-2 N_{n}, 2 N_{n}\right]} \frac{\tilde{\rho}_{n}(d u)}{(x-u)^{2}+h_{n}^{2}}, \quad x \in \mathbb{R} .
$$

From (4.37) it follows

$$
\begin{aligned}
& \left|\int_{\mathbb{R}} e^{i t x} \Im R_{n}\left(\left(\tilde{Z}_{n}\right)^{(-1)}\left(x+i h_{n}\right)\right) d x\right| \leq \int_{\mathbb{R}}\left|R_{n}\left(\left(\tilde{Z}_{n}\right)^{(-1)}\left(x+i h_{n}\right)\right)\right| d x \\
& \leq c(\mu) n^{3} N_{n}^{2} \varepsilon_{n}^{*} \int_{\mathbb{R}} d x \int_{\left[-2 N_{n}, 2 N_{n}\right]} \frac{\tilde{\rho}_{n}(d u)}{(x-u)^{2}+h_{n}^{2}} \leq c(\mu) n^{3} N_{n}^{2} \varepsilon_{n}^{*} h_{n}^{-1}, \quad t \in \mathbb{R} .
\end{aligned}
$$


Denote $V_{n}(u):=\tilde{\mu}_{n}((-\infty, u))-\tilde{\nu}_{n}((-\infty, u)), u \in \mathbb{R}$. Integrating by parts, we obtain

$$
\begin{aligned}
& \left|\Im \int_{\left[-2 n N_{n}, 2 n N_{n}\right]} \frac{\left(\tilde{\mu}_{n}-\tilde{\nu}_{n}\right)(d u)}{\tilde{Z}_{n}^{(-1)}\left(x+i h_{n}\right)-u}\right| \\
& =\left|\int_{\left[-2 n N_{n}, 2 n N_{n}\right]} \frac{\Im \tilde{Z}_{n}^{(-1)}\left(x+i h_{n}\right)\left(\tilde{\mu}_{n}-\tilde{\nu}_{n}\right)(d u)}{\left(\Re \tilde{Z}_{n}^{(-1)}\left(x+i h_{n}\right)-u\right)^{2}+\left(\Im\left(\tilde{Z}_{n}\right)^{(-1)}\left(x+i h_{n}\right)\right)^{2}}\right| \\
& \leq 2 \int_{\left[-2 n N_{n}, 2 n N_{n}\right]} \frac{\Im \tilde{Z}_{n}^{(-1)}\left(x+i h_{n}\right)\left|\Re \tilde{Z}_{n}^{(-1)}\left(x+i h_{n}\right)-u\right|\left|V_{n}(u)\right| d u}{\left(\left(\Re \tilde{Z}_{n}^{(-1)}\left(x+i h_{n}\right)-u\right)^{2}+\left(\Im \tilde{Z}_{n}^{(-1)}\left(x+i h_{n}\right)\right)^{2}\right)^{2}} \\
& \leq \int_{\left[-2 n N_{n}, 2 n N_{n}\right]} \frac{\left|V_{n}(u)\right| d u}{\left(\Re \tilde{Z}_{n}^{(-1)}\left(x+i h_{n}\right)-u\right)^{2}+\left(\Im \tilde{Z}_{n}^{(-1)}\left(x+i h_{n}\right)\right)^{2}} .
\end{aligned}
$$

In view of (4.19) and (4.34), (4.35), we deduce from this estimate the following inequality

$$
\left|\Im \int_{\left[-2 n N_{n}, 2 n N_{n}\right]} \frac{\left(\tilde{\mu}_{n}-\tilde{\nu}_{n}\right)(d u)}{\tilde{Z}_{n}^{(-1)}\left(x+i h_{n}\right)-u}\right| \leq\left\{\begin{array}{lll}
c(\mu) n N_{n} \varepsilon_{n}^{*} & \text { for } & |x| \leq 5 n N_{n} \\
c(\mu) n^{2} N_{n} \varepsilon_{n}^{*} /\left(1+x^{2}\right) & \text { for } & |x|>5 n N_{n}
\end{array}\right.
$$

which implies the upper bound

$$
\left|\int_{\mathbb{R}} e^{i t x} \Im \int_{\left[-2 n N_{n}, 2 n N_{n}\right]} \frac{\left(\tilde{\mu}_{n}-\tilde{\nu}_{n}\right)(d u)}{\tilde{Z}_{n}^{(-1)}\left(x+i h_{n}\right)-u} d x\right| \leq c(\mu) n^{2} N_{n}^{2} \varepsilon_{n}^{*}, \quad t \in \mathbb{R} .
$$

It remains to note that

$$
\int_{\mathbb{R}} e^{i t x} \Im \int_{\left[-2 N_{n}, 2 N_{n}\right]} \frac{\left(\tilde{\mu}-\tilde{\rho}_{n}\right)(d u)}{x-u+i h_{n}} d x=e^{-h_{n}|t|}\left(\varphi(t ; \tilde{\mu})-\varphi\left(t ; \tilde{\rho}_{n}\right)\right), \quad t \in \mathbb{R}
$$

where $\varphi(t ; \tilde{\mu})$ and $\left.\varphi\left(t ; \tilde{\rho}_{n}\right)\right)$ are characteristic functions of the probability measures $\tilde{\mu}$ and $\tilde{\rho}_{n}$, respectively. Recalling (4.19), we finally conclude from (4.36), (4.38)-(4.40) that, for every fixed $v>0$,

$$
\left.\int_{[0, v]} \mid \varphi(t ; \tilde{\mu})-\varphi\left(t ; \tilde{\rho}_{n}\right)\right) \mid d t \leq c(\mu) n^{5 / 2} N_{n}^{2} e^{v h_{n}} \varepsilon_{n}^{*}
$$

Since $\varepsilon_{n}^{*} \leq 5 n^{3} \varepsilon_{n}^{3 / 13}$, we see that, for $0<v<\frac{1}{260} \frac{\beta^{\prime}}{\sqrt{m_{2}(\mu)}}$

$$
n^{5 / 2} N_{n}^{2} e^{v h_{n}} \varepsilon_{n}^{*} \leq 5 n^{11 / 2} \varepsilon_{n}^{1 / 13} e^{10 v \sqrt{m_{2}(\mu)} \sqrt{n}} \leq 5 n^{11 / 2} \varepsilon_{n}^{1 / 26}
$$


Note that the right-hand side of this inequality tends to zero as $n \rightarrow \infty$. Since $\mu\left(\mathbb{R} \backslash\left[-2 N_{n}, 2 N_{n}\right]\right) \leq \beta_{3}(\mu) /\left(2 N_{n}\right)^{3}$, we obtain from (4.41) the upper bound

$$
\begin{aligned}
\int_{[0, v]}\left(1-\Re \varphi\left(t ; \tilde{\rho}_{n}\right)\right) d t & \leq \int_{[0, v]}(1-\Re \varphi(t ; \tilde{\mu})) d t+c(\mu) n^{11 / 2} \varepsilon_{n}^{1 / 26} \\
& \leq \int_{[0, v]}(1-\Re \varphi(t ; \mu)) d t+c(\mu) n^{11 / 2} \varepsilon_{n}^{1 / 26}
\end{aligned}
$$

for every fixed $0<v<\frac{1}{260} \frac{\beta^{\prime}}{\sqrt{m_{2}(\mu)}}$.

Now we shall show that in the sequence $\left\{\tilde{\rho}_{n}\right\}$ we can choose a weakly convergent subsequence. In order to prove this, we shall verify that for any $\varepsilon>0$ there exists $N(\varepsilon)>0$ such that

$$
\left.\sup _{n} \tilde{\rho}_{n}(\mathbb{R} \backslash[-N(\varepsilon)), N(\varepsilon)]\right) \leq \varepsilon .
$$

It is obvious that (4.43) follows from Proposition 3.6 and (4.42). We will denote a weakly convergent subsequence of measures $\left\{\tilde{\rho}_{n}\right\}$ by $\left\{\tilde{\rho}_{n}\right\}$ again. We see that $\left\{\tilde{\rho}_{n}\right\}$ converges weakly to an probability measure $\rho$ and, by (4.41),

$$
\varphi(t ; \mu)=\varphi(t ; \rho) \quad \text { for } \quad t \in\left[-\frac{\beta}{300 \sqrt{m_{2}(\mu)}}, \frac{\beta}{300 \sqrt{m_{2}(\mu)}}\right] .
$$

. Thus, the theorem is proved.

\section{Probability measures $\mu$ With exponential DeCrease in $\Delta\left(\mu^{n \boxplus}, \mathbf{D}^{\boxplus}\right)$}

The aim of this section is to prove Theorem 2.9. In the sequel we need some results of the classical moment problem (see[1]). R. Nevanlinna established a complete description of all solutions for the indeterminate Hamburger moment problem which we will briefly recall here. We use $P_{k}(z)$ and $Q_{k+1}(z)\left(Q_{0}(z) \equiv 0\right)$, to denote orthogonal polynomials of degree $k$ of the first and second kinds, respectively, that corresponds to the indeterminate moment problem $\left\{m_{k}(\mu)\right\}_{k=0}^{\infty}$. The polynomials $P_{k}(z)$ and $Q_{k}(z)$ are real-valued on the real line, and for them, the series $\sum_{k=0}^{\infty}\left|P_{k}(z)\right|^{2}$ and $\sum_{k=0}^{\infty}\left|Q_{k}(z)\right|^{2}$ converge almost everywhere in C. Define the four functions

$$
\begin{aligned}
& A(z)=z \sum_{k=0}^{\infty} Q_{k}(0) Q_{k}(z), \quad B(z)=-1+z \sum_{k=0}^{\infty} Q_{k}(0) P_{k}(z), \\
& C(z)=1+z \sum_{k=0}^{\infty} P_{k}(0) Q_{k}(z), \quad E(z)=z \sum_{k=0}^{\infty} P_{k}(0) P_{k}(z) .
\end{aligned}
$$

As Riesz M. has proved (see[1], Chapter 3), the functions $A(z), B(z), C(z)$, and $E(z)$ are entire functions of no more than minimal type of order 1. It is known that the zeros of the entire functions $A(z) t-C(z)$ and $B(z) t-E(z)$ are real alternate and of multiplicity one for any real $t$. 
According to Nevanlinna's theorem (see [1]), the formula

$$
\int_{\mathbb{R}} \frac{\mu(d u)}{u-z}=-\frac{A(z) f(z)-C(z)}{B(z) f(z)-E(z)}
$$

establishes a one-to-one relationship between the set of all solutions $\mu$ of the moments problem under consideration and the set of all functions $f(z)$ of the Nevanlinna class, completed by the constant $\infty$. Finite real constants $t$ belong to this class by definition. In this paper we are interested only in the solutions of the moment problem that corresponds to the functions $f(z) \equiv t$. We denote these solutions by $\mu_{t}, t \in \mathbb{R}$. These probability measures $\mu_{t}$ are discrete and have growth points at the zeros of the functions $B(z) t-E(z)$.

Consider the sequence $\alpha_{s}=\int_{\mathbb{R}} x^{s} e^{-2|x| / L(|x|)} d x, s=0,1, \ldots$, where $L(x) \geq 1, x \geq 0$, is a slowly varying function such that $\int_{\mathbb{R}_{+}} \frac{d x}{(1+x) L(x)}<\infty$. Without loss of generality we assume that $L(x)$ has continuous derivative. For simplicity we assume $\alpha_{0}=1$ as well. By a result of Krein (see [1], Ch. 2), the sequence $\left\{\alpha_{s}\right\}_{s=0}^{\infty}$ generates an indeterminate moment problem.

Now introduce a symmetric $\boxplus$-infinitely divisible probability measure $\mu$ with free cumulants $\alpha_{s}(\mu)=\alpha_{s}, s=1,2, \ldots$ We see that that the sequence $\left\{m_{s}(\mu)\right\}_{s=0}^{\infty}$ generates an indeterminate moment problem as well. Consider symmetric probability measures $\frac{1}{2}\left(\mu_{t}+\bar{\mu}_{t}\right), t \in \mathbb{R}$. These measures are discrete and therefore are not $\boxplus$-infinitely divisible.

First evaluate the cumulants $\alpha_{2 s}(\mu), s=1, \ldots$

Since $L(x)$ from Theorem 2.9 satisfies the functional equation $L(x) \sim L(x / L(x))$ as $x \rightarrow \infty$, we see from [25], p. 48-49, that

$$
L(x) \sim L(x / L(x)) \sim L(x L(x)) \quad \text { as } \quad x \rightarrow \infty .
$$

Lemma 5.1. The following inequalities hold

$$
\alpha_{2 s}(\mu) \leq c(3 s L(s))^{2 s} e^{-3 s / 4}, \quad s \in \mathbb{N}
$$

with some constant $c$ depending on $L$ only.

Proof. It is well-known that $x L^{\prime}(x) / L(x)=o(1)$ as $x \rightarrow \infty$, see [25]. Using this relation and (5.2) we conclude that the function $x / L(x)$ increases monotonously as $x \rightarrow \infty$ and the equation $x / L(x)=2 s$ has a unique solution $x_{s}$ such that $x_{s} \sim 2 s L(2 s) \sim 2 s L(s)$ as $s \rightarrow \infty$. Hence solutions $y_{s}$ of the equations

$$
\frac{x}{L(x)}\left(1-\frac{x L^{\prime}(x)}{L(x)}\right)=2 s
$$


satisfy the inequality $s L(s) \leq y_{s} \leq 3 s L(s)$ for sufficiently large $s \geq s_{0} \geq 1$. It remains to note that the inequality

$$
\begin{aligned}
\int_{\mathbb{R}} x^{2 s} e^{-2|x| / L(|x|)} d x & \leq \max _{y_{s}}\left(y_{s}^{2 s} e^{-y_{s} / L\left(y_{s}\right)}\right) \int_{\mathbb{R}} e^{-|x| / L(|x|)} d x \\
& \leq c(3 s L(s))^{2 s} e^{-s L(s) / L(s / L(s))} \leq c(3 s L(s))^{2 s} e^{-3 s / 4}
\end{aligned}
$$

holds for $s \geq s_{0}$ with some constant $c$ depending on $L$ only. The lemma is proved.

Evaluate moments $m_{s}(\mu), s=2,3, \ldots$

Lemma 5.2. The inequalities hold

$$
\alpha_{2 s} \leq m_{2 s}(\mu) \leq 4^{2 s} \alpha_{2 s}, \quad s \in \mathbb{N} .
$$

Proof. Since $\alpha_{2 j-1}(\mu)=\alpha_{2 j-1}=0$ and $\alpha_{2 j}(\mu)=\alpha_{2 j}>0$ for $j=1, \ldots$, the left inequality in (5.4) obviously follows from (3.12). Now we obtain from (3.11) and (3.12)

$$
m_{2 s}(\mu) \leq \frac{1}{2 s} \sum_{l=1}^{2 s}\left(\begin{array}{c}
2 s \\
l
\end{array}\right)\left(\begin{array}{c}
2 s \\
l-1
\end{array}\right) \prod_{j=1}^{l} \alpha_{\left|V_{j}\right|}(\mu)=\frac{1}{2 s} \sum_{l=1}^{2 s}\left(\begin{array}{c}
2 s \\
l
\end{array}\right)\left(\begin{array}{c}
2 s \\
l-1
\end{array}\right) \prod_{j=1}^{l} \alpha_{\left|V_{j}\right|}, \quad s \in \mathbb{N} .
$$

By Lyapunov's inequality, $\alpha_{\left|V_{j}\right|} \leq\left(\alpha_{2 s}\right)^{\left|V_{j}\right| /(2 s)}$. Therefore we have from (15.5)

$$
m_{2 s}(\mu) \leq \alpha_{2 s} \frac{1}{2 s} \sum_{l=1}^{2 s}\left(\begin{array}{c}
2 s \\
l
\end{array}\right)\left(\begin{array}{c}
2 s \\
l-1
\end{array}\right) \leq \frac{\alpha_{2 s}}{2 s} 4^{2 s}
$$

The right inequality in (5.4) follows immediately from this estimate.

Using Lemma 5.2 we estimate tails of the measures $\mu$ and $\mu_{t}$.

Lemma 5.3. The following upper bounds hold

$$
\mu(\{|x| \geq N\}) \leq c(\mu) e^{-N /(48 L(N))}, \quad \mu_{t}(\{|x| \geq N\}) \leq c(\mu) e^{-N /(48 L(N))}, \quad N \geq 1 .
$$

Proof. By Lemmas 5.1 and 5.2 we have

$$
m_{2 s}(\mu) \leq c(12 s L(s))^{2 s} e^{-3 s / 4}, \quad s \in \mathbb{N},
$$

with some constant $c$ depending on $L$. It is easy follows from this bound that

$$
\mu(\{|x|>12 s L(s)\}) \leq c e^{-3 s / 4}, \quad s \in \mathbb{N} .
$$

This inequality holds obviously for all positive $s \geq 1$. Using (5.2) we deduce from the last inequality the following estimate

$$
\mu\left(\left\{|x|>12 s^{*}\right\}\right) \leq c e^{-s^{*} / 2 L\left(s^{*}\right)}
$$

for all positive sufficiently large $s^{*}=s L(s), s \geq s_{0}$. The assertion of the lemma for the measure $\mu$ follows immediately from this upper bound. One can prove the lemma for the measures $\mu_{t}$ in the same way. 
Introduce the segment $\left[-N_{n}, N_{n}\right]$ with $N_{n}:=\sqrt{n} / \tilde{c}(\mu)$ with sufficiently large constant $\tilde{c}(\mu)>0$ and consider probability measures $\tilde{\mu}$ and $\tilde{\mu}_{t}$ which are truncated on this segment. By Lemma 5.3 and Proposition 3.5, we have

$$
\Delta\left(\mu_{t}^{n \boxplus}, \mu^{n \boxplus}\right) \leq \Delta\left(\tilde{\mu}_{t}^{n \boxplus}, \tilde{\mu}^{n \boxplus}\right)+c(\mu) n e^{-N_{n} /\left(48 L\left(N_{n}\right)\right)}, \quad n \in \mathbb{N},
$$

where $c(\mu)>0$ does not depend on $\tilde{c}(\mu)$. In the sequel constants $c(\mu)$ do not depend on $\tilde{c}(\mu)$ as well.

In addition moments the probability measures $\tilde{\mu}$ and $\tilde{\mu}_{t}$ are close in the following sense.

Lemma 5.4. The following upper bounds hold

$$
\left|m_{2 s}(\tilde{\mu})-m_{2 s}\left(\tilde{\mu}_{t}\right)\right| \leq c(\mu) N_{n}^{2 s} e^{-N_{n} /\left(96 L\left(N_{n}\right)\right)}, \quad s=1, \ldots,\left[N_{n} /\left(200 L\left(N_{n}\right)\right)\right] .
$$

Proof. By the definition of $\tilde{\mu}$ and $\tilde{\mu}_{t}$, we have

$$
\left|m_{2 s}(\tilde{\mu})-m_{2 s}\left(\tilde{\mu}_{t}\right)\right| \leq \int_{|x| \geq N_{n}} x^{2 s} \mu(d x)+\int_{|x| \geq N_{n}} x^{2 s} \mu_{t}(d x) .
$$

The function $x^{2 s-1} \exp \{-x /(96 L(x))\}, x \geq N_{n}$, is decreasing, therefore integrating by parts and using Lemma [5.3, we see that

$$
\begin{aligned}
\int_{|x| \geq N_{n}} x^{2 s} \mu(d x) & \leq N_{n}^{2 s} \mu\left(\left\{|x| \geq N_{n}\right\}\right)+2 s \int_{N_{n}}^{\infty} x^{2 s-1} \mu(\{|x| \geq x\}) d x \\
& \leq c(\mu) N_{n}^{2 s} e^{-N_{n} /\left(48 L\left(N_{n}\right)\right)}+2 c(\mu) s \int_{N_{n}}^{\infty} x^{2 s-1} e^{-x /(48 L(x))} d x \\
& \leq c(\mu) N_{n}^{2 s} e^{-N_{n} /\left(96 L\left(N_{n}\right)\right)} .
\end{aligned}
$$

In the same way we obtain the bound

$$
\int_{|x| \geq N_{n}} x^{2 s} \mu_{t}(d x) \leq c(\mu) N_{n}^{2 s} e^{-N_{n} /\left(96 L\left(N_{n}\right)\right)} .
$$

The last two bounds imply the assertion of the lemma.

As before in Section $4 G_{\tilde{\mu}^{n \boxplus}}(z)=G_{\tilde{\mu}}\left(\tilde{Z}_{n}(z)\right)$ and $G_{\tilde{\mu}_{t} n \boxplus}(z)=G_{\tilde{\mu}_{t}}\left(\tilde{W}_{n}(z)\right)$, where $\tilde{Z}_{n}(z)$ and $\tilde{W}_{n}(z)$ belong to the class $\mathcal{F}$ and satisfy the functional equations (4.21) and (4.23) with $\tilde{\rho_{n}}=\tilde{\mu}_{t}$, respectively. By Lemma 3.9 , we conclude that $\tilde{Z}_{n}(z)$ and $\tilde{W}_{n}(z)$ admit representations (4.22) and (4.24), respectively, where the measures $\tilde{\sigma}_{n}$ and $\tilde{\tau}_{n}$ satisfy the conditions, for some $c(\mu)>0$,

$\tilde{\sigma}_{n}(\mathbb{R})=\tilde{\sigma}_{n}\left(\left[-c(\mu)(n-1) N_{n}, c(\mu)(n-1) N_{n}\right]\right), \tilde{\tau}_{n}(\mathbb{R})=\tilde{\tau}_{n}\left(\left[-c(\mu)(n-1) N_{n}, c(\mu)(n-1) N_{n}\right]\right)$.

Therefore, for $u \in\left[-N_{n}, N_{n}\right]$,

$$
\frac{1}{u-\tilde{Z}_{n}(z)}=\int_{\mathbb{R}} \frac{\zeta(u ; d s)}{s-z} \quad \text { and } \quad \frac{1}{u-\tilde{W}_{n}(z)}=\int_{\mathbb{R}} \frac{\xi(u ; d s)}{s-z}, \quad z \in \mathbb{C}^{+},
$$

where $\zeta(u ; d s)$ and $\xi(u ; d s)$ are probability measures such that $\zeta\left(u ;\left[-c(\mu)(n-1) N_{n}, c(\mu)(n-\right.\right.$ 1) $\left.\left.N_{n}\right]\right)=1$ and $\xi\left(u ;\left[-c(\mu)(n-1) N_{n}, c(\mu)(n-1) N_{n}\right]\right)=1$ with some $c(\mu)>0$. Hence 
the probability measures $\tilde{\mu}^{n \boxplus}$ and $\tilde{\mu}_{t}^{n \boxplus}$ are supported on the segment $\left[-c(\mu)(n-1) N_{n}, c(\mu)(n-\right.$ 1) $\left.N_{n}\right]$.

Recall the definition of the domains $D_{n}\left(\tilde{\mu_{t}}\right)=\left\{z=x+i y, x, y \in \mathbb{R}: y>\tilde{y}_{t}(x)\right\}$ and $D_{n}(\tilde{\mu})=\{z=x+i y, x, y \in \mathbb{R}: y>\tilde{y}(x)$. By Lemma 3.4 we have the following lower bounds

$$
\left|\tilde{Z}_{n}(z)\right| \geq c(\mu) \sqrt{n}, \quad\left|\tilde{W}_{n}(z)\right| \geq c(\mu) \sqrt{n}, \quad z \in \mathbb{C}^{+}, \quad n \in \mathbb{N} .
$$

Using (4.21) and (4.23) with $\tilde{\rho}_{n}=\tilde{\mu_{t}}$ we obtain the relation, for $z \in \mathbb{C}^{+}$,

$$
-(n-1)\left(G_{\tilde{\mu}}\left(\tilde{Z}_{n}(z)\right)-G_{\tilde{\mu_{t}}}\left(\tilde{W}_{n}(z)\right)\right)=n\left(\tilde{Z}_{n}(z)-\tilde{W}_{n}(z)\right) G_{\tilde{\mu}}\left(\tilde{Z}_{n}(z)\right) G_{\tilde{\mu_{t}}}\left(\tilde{W}_{n}(z)\right) .
$$

Since $G_{\tilde{\mu}}\left(\tilde{Z}_{n}(z)\right)=\int_{\left[-N_{n}, N_{n}\right]} \frac{\tilde{\mu}(d u)}{\tilde{Z}_{n}(z)-u}$ for $z \in \mathbb{C}^{+}$, by $(\underline{5.8})$, we see that $\mid G_{\tilde{\mu}}\left(\tilde{Z}_{n}(z) \mid \leq\right.$ $c(\mu) / N_{n}$ for $\Im z \geq 0$. The same bound holds for $\mid G_{\tilde{\mu_{t}}}\left(\tilde{W}_{n}(z) \mid\right.$. Therefore we conclude from (5.9) that

$$
\left|G_{\tilde{\mu}}\left(\tilde{Z}_{n}(z)\right)-G_{\tilde{\mu_{t}}}\left(\tilde{W}_{n}(z)\right)\right| \leq \frac{c(\mu)}{N_{n}^{2}}\left|\tilde{Z}_{n}(z)-\tilde{W}_{n}(z)\right|, \quad \Im z \geq 0 .
$$

Now we need to estimate the closeness of the functions $\tilde{Z}_{n}(z)$ and $\tilde{W}_{n}(z)$.

Lemma 5.5. The following upper bounds hold

$$
\left|\tilde{Z}_{n}(z)-\tilde{W}_{n}(z)\right| \leq c(\mu) e^{-c(\mu) \sqrt{n} / L(\sqrt{n})}, \quad \Im z \geq 0 .
$$

In order to prove this lemma we need some auxiliary results.

Lemma 5.6. The upper bound holds, for $\Im z \geq 0,|z| \geq 100 N_{n}$,

$$
\left|\tilde{Z}_{n}{ }^{(-1)}(z)-\tilde{W}_{n}{ }^{(-1)}(z)\right| \leq c(\mu)(n-1) N_{n} e^{-N_{n} /\left(96 L\left(N_{n}\right)\right)} .
$$

Proof. Note that, for $z \in \mathbb{C}^{+}$,

$$
\tilde{Z}_{n}{ }^{(-1)}(z)-\tilde{W}_{n}{ }^{(-1)}(z)=(n-1)\left(F_{\tilde{\mu}_{t}}(z)-F_{\tilde{\mu}}(z)\right)=\frac{n-1}{G_{\tilde{\mu}_{t}}(z) G_{\tilde{\mu}}(z)}\left(G_{\tilde{\mu}}(z)-G_{\tilde{\mu}_{t}}(z)\right) .
$$

On the other hand we see that, for $l_{1}=\left[N_{n} /\left(200 L\left(N_{n}\right)\right)\right]$,

$$
\begin{aligned}
\left.G_{\tilde{\mu}}(z)-G_{\tilde{\mu}_{t}}(z)\right) & =\frac{m_{2}(\tilde{\mu})-m_{2}\left(\tilde{\mu}_{t}\right)}{z^{3}}+\cdots+\frac{m_{2\left(l_{1}-1\right)}(\tilde{\mu})-m_{2\left(l_{1}-1\right)}\left(\tilde{\mu}_{t}\right)}{z^{2 l_{1}-1}} \\
& +\frac{1}{z^{2 l_{1}}} \int_{\mathbb{R}} \frac{u^{2 l_{1}}\left(\tilde{\mu}-\tilde{\mu}_{t}\right)(d u)}{z-u} .
\end{aligned}
$$

By Lemma 5.4 and (5.6), we conclude that

$$
\begin{aligned}
& \left|G_{\tilde{\mu}}(z)-G_{\tilde{\mu}_{t}}(z)\right| \leq \frac{c(\mu)}{|z|^{2}} e^{-N_{n} /\left(96 L\left(N_{n}\right)\right)} \sum_{s=1}^{l_{1}} \frac{N_{n}^{2 s}}{\left(100 N_{n}\right)^{2 s-1}}+\frac{c(\mu)}{|z|^{2}} \frac{m_{2 l_{1}}(\mu)}{\left(100 N_{n}\right)^{2 l_{1}-1}} \\
& \leq \frac{c(\mu)}{|z|^{2}}\left(N_{n} e^{-N_{n} /\left(96 L\left(N_{n}\right)\right)}+N_{n}\left(\frac{12 l_{1} L\left(l_{1}\right)}{100 N_{n}}\right)^{2 l_{1}}\right) \leq \frac{c(\mu)}{|z|^{2}} N_{n} e^{-N_{n} /\left(96 L\left(N_{n}\right)\right)} .
\end{aligned}
$$

Applying this bound and the lower bounds $\left|G_{\tilde{\mu}_{t}}(z)\right| \geq c(\mu) /|z|,\left|G_{\tilde{\mu}}(z)\right| \geq c(\mu) /|z|$, for $|z| \geq 2 N_{n}$, to (5.13) we arrive at the assertion of the lemma. 
Lemma 5.7. The following upper bound holds, for $z \in \mathbb{C}^{+},|z| \geq 100 N_{n}$,

$$
\frac{1}{\Im z}\left|\Im\left(\frac{1}{G_{\tilde{\mu}}(z)}-\frac{1}{G_{\tilde{\mu}_{t}}(z)}\right)\right| \leq c(\mu) N_{n} e^{-N_{n} /\left(96 L\left(N_{n}\right)\right)} .
$$

Proof. We will use the following simple estimate

$$
\begin{aligned}
& \frac{1}{\Im z}\left|\Im\left(\frac{1}{G_{\tilde{\mu}}(z)}-\frac{1}{G_{\tilde{\mu}_{t}}(z)}\right)\right| \leq \frac{1}{\Im z}\left|\Im\left(G_{\tilde{\mu}}(z)-G_{\tilde{\mu}_{t}}(z)\right)\right| \frac{1}{\left.\mid G_{\tilde{\mu}}(z) G_{\tilde{\mu}_{t}}(z)\right) \mid} \\
& +\left|G_{\tilde{\mu}}(z)-G_{\tilde{\mu}_{t}}(z)\right| \frac{1}{\Im z}\left(\frac{\Im G_{\tilde{\mu}}(z)}{\left|G_{\tilde{\mu}}(z)\right|^{2}} \frac{1}{\left|G_{\tilde{\mu}_{t}}(z)\right|}+\frac{\Im G_{\tilde{\mu}_{t}}(z)}{\left|G_{\tilde{\mu}_{t}}(z)\right|^{2}} \frac{1}{\left|G_{\tilde{\mu}}(z)\right|}\right) .
\end{aligned}
$$

Using the formula (5.24) and repeating the argument of the proof of Lemma 5.6, we see that the first term of the sum on the right hand-side of (5.16) does not exceed $c(\mu) N_{n} e^{-N_{n} /\left(96 L\left(N_{n}\right)\right)}$. Noting that $\frac{1}{\Im z}\left|\Im G_{\tilde{\mu}}(z)\right| \leq \frac{4}{|z|^{2}}$ and $\frac{1}{\Im z}\left|\Im G_{\tilde{\mu}_{t}}(z)\right| \leq \frac{4}{|z|^{2}}$, for $|z| \geq$ $2 N_{n}$, and using (5.15), we obtain that the second term in the sum on the right hand-side of (5.16) does not exceed the quantity $\frac{c(\mu)}{|z|} N_{n} e^{-N_{n} /\left(96 L\left(N_{n}\right)\right)}$. The assertion of the lemma follows from the last two bounds.

Recalling Proposition 3.1 we see that

$$
\tilde{Z}_{n}^{(-1)}(z)=z-(n-1) \int_{\left[-N_{n}, N_{n}\right]} \frac{\tilde{\sigma}(d u)}{u-z} \quad \text { and } \quad \tilde{W}_{n}^{(-1)}(z)=z-(n-1) \int_{\left[-N_{n}, N_{n}\right]} \frac{\tilde{\sigma}_{t}(d u)}{u-z},
$$

where $\tilde{\sigma}$ and $\tilde{\sigma}_{t}$ are non negative finite measures. Denote by $M_{n}(\tilde{\mu}) \geq N_{n}$ and $M_{n}\left(\tilde{\mu}_{t}\right) \geq N_{n}$ positive numbers satisfying the equations

$$
\int_{-\left[N_{n}, N_{n}\right]} \frac{\tilde{\sigma}(d u)}{\left(u+M_{n}(\tilde{\mu})\right)^{2}}=\frac{1}{n-1} \text { and } \int_{\left[-N_{n}, N_{n}\right]} \frac{\tilde{\sigma}_{t}(d u)}{\left(u+M_{n}\left(\tilde{\mu}_{t}\right)\right)^{2}}=\frac{1}{n-1},
$$

respectively. Without loss of generality we assume that $M_{n}(\tilde{\mu}) \geq M_{n}\left(\tilde{\mu}_{t}\right)$. Now we define a Jordan curve $\gamma_{n}(\tilde{\mu})=\left\{y=y_{n}(x ; \tilde{\mu}), x \in \mathbb{R}\right\}$, where the function $y_{n}(x ; \tilde{\mu})$ is the solution of the functional equation

$$
\int_{\left[-N_{n}, N_{n}\right]} \frac{\tilde{\sigma}(d u)}{(u-x)^{2}+y_{n}(x ; \tilde{\mu})^{2}}=\frac{1}{n-1}
$$

for $x \in\left[-M_{n}(\tilde{\mu}), M_{n}(\tilde{\mu})\right]$. For $|x|>M_{n}(\tilde{\mu}) y_{n}(x ; \tilde{\mu})=0$. In the same way we define the curve $\gamma_{n}\left(\tilde{\mu}_{t}\right)=\left\{y=y_{n}\left(x ; \tilde{\mu}_{t}\right), x \in \mathbb{R}\right\}$.

Lemma 5.8. The following bound holds

$$
\left|y_{n}(x ; \tilde{\mu})-y_{n}\left(x ; \tilde{\mu}_{t}\right)\right| \leq c(\mu) e^{-N_{n} /\left(200 L\left(N_{n}\right)\right)}, \quad x \in \mathbb{R} .
$$


Proof. Assume that $-M_{n}\left(\tilde{\mu}_{t}\right)<x \leq 0$. In this case $y_{n}(x ; \tilde{\mu})>0$ and $y_{n}\left(x ; \tilde{\mu}_{t}\right)>0$, and we have the relation

$$
\begin{gathered}
\left(y_{n}\left(x ; \tilde{\mu}_{t}\right)^{2}-y_{n}(x ; \tilde{\mu})^{2}\right) \int_{\left[-N_{n}, N_{n}\right]} \frac{\tilde{\sigma}(d u)}{\left((u-x)^{2}+y_{n}(x ; \tilde{\mu})^{2}\right)\left((u-x)^{2}+y_{n}\left(x ; \tilde{\mu}_{t}\right)^{2}\right)} \\
=\frac{1}{y_{n}\left(x ; \tilde{\mu}_{t}\right)} \Im\left(\frac{1}{G_{\tilde{\mu}_{t}}\left(x+y_{n}\left(x ; \tilde{\mu}_{t}\right)\right)}-\frac{1}{G_{\tilde{\mu}}\left(x+y_{n}\left(x ; \tilde{\mu}_{t}\right)\right)}\right) .
\end{gathered}
$$

Since $\left.x^{2}+y_{n}\left(x ; \tilde{\mu}_{t}\right)\right)^{2} \geq c(\mu) n$ we obtain, using Lemma 5.7 ,

$$
\begin{aligned}
& \mid y_{n}\left(x ; \tilde{\mu}_{t}\right)-\left.y_{n}(x ; \tilde{\mu})\right|^{2} \int_{\left[-N_{n}, N_{n}\right]} \frac{\tilde{\sigma}(d u)}{\left((u-x)^{2}+y_{n}(x ; \tilde{\mu})^{2}\right)\left((u-x)^{2}+y_{n}\left(x ; \tilde{\mu}_{t}\right)^{2}\right)} \\
& \leq c(\mu) \sqrt{n} e^{-N_{n} /\left(96 L\left(N_{n}\right)\right)} .
\end{aligned}
$$

Since the integral in this inequality is greater or equal to $c(\mu) / n^{2}$, we finally get the upper bound $\left|y_{n}\left(x ; \tilde{\mu}_{t}\right)-y_{n}(x ; \tilde{\mu})\right| \leq c(\mu) e^{-N_{n} /\left(200 L\left(N_{n}\right)\right)}$ for $-M_{n}\left(\tilde{\mu}_{t}\right)<x \leq 0$. Since $y_{n}\left(-M_{n}\left(\tilde{\mu}_{t}\right) ; \tilde{\mu}_{t}\right)=0$, we have $y_{n}\left(-\bar{M}_{n}\left(\tilde{\mu}_{t}\right) ; \tilde{\mu}\right) \leq c(\mu) e^{-N_{n} /\left(200 L\left(N_{n}\right)\right)}$. The function $y_{n}(x ; \tilde{\mu})$ is increasing for $-M_{n}(\tilde{\mu}) \leq x \leq-M_{n}\left(\tilde{\mu}_{t}\right)$, hence $y_{n}(x ; \tilde{\mu}) \leq c(\mu) e^{-N_{n} /\left(200 L\left(N_{n}\right)\right)}$ for such $x$. The same arguments hold for $x>0$. The lemma is proved.

Lemma 5.9. The function $y_{n}\left(x ; \tilde{\mu}_{t}\right)$ is differentiable for $|x|<M_{n}\left(\tilde{\mu}_{t}\right)$ with the derivative satisfying the relation

$$
y_{n}\left(x ; \tilde{\mu}_{t}\right) y_{n}^{\prime}\left(x ; \tilde{\mu}_{t}\right)=\int_{-\left[N_{n}, N_{n}\right]} \frac{(u-x) \tilde{\sigma}_{t}(d u)}{\left((u-x)^{2}+y_{n}\left(x ; \tilde{\mu}_{t}\right)^{2}\right)^{2}} / \int_{\left[-N_{n}, N_{n}\right]} \frac{\tilde{\sigma}_{t}(d u)}{\left((u-x)^{2}+y_{n}\left(x ; \tilde{\mu}_{t}\right)^{2}\right)^{2}} .
$$

Proof. The proof is simple and we omit it.

Now we prove Lemma 5.5.

Proof. Consider the functions

$$
\begin{aligned}
f(x) & =Z_{n}^{(-1)}\left(x+i y_{n}(x ; \tilde{\mu})\right), \quad|x|<M_{n}(\tilde{\mu}), \\
f_{t}(x) & =W_{n}^{(-1)}\left(x+i y_{n}\left(x ; \tilde{\mu}_{t}\right)\right),|x|<M_{n}\left(\tilde{\mu}_{t}\right) .
\end{aligned}
$$

We see that, for $|x|<M_{n}\left(\tilde{\mu}_{t}\right)$,

$$
\begin{aligned}
f_{t}^{\prime}(x) & =1+(n-1) \int_{\left[-N_{n}, N_{n}\right]} \frac{\tilde{\sigma}_{t}(d u)}{(u-x)^{2}+y_{n}\left(x ; \tilde{\mu}_{t}\right)^{2}} \\
& +(n-1) \int_{\left[-N_{n}, N_{n}\right]}(u-x) \frac{-2(u-x)+2 y_{n}\left(x ; \tilde{\mu}_{t}\right) y_{n}^{\prime}\left(x ; \tilde{\mu}_{t}\right)}{\left.\left((u-x)^{2}+y_{n}\left(x ; \tilde{\mu}_{t}\right)^{2}\right)\right)^{2}} \tilde{\sigma}_{t}(d u) \\
& =2(n-1)\left(\int_{\left[-N_{n}, N_{n}\right]} \frac{y_{n}\left(x ; \tilde{\mu}_{t}\right)^{2} \tilde{\sigma}_{t}(d u)}{\left.(u-x)^{2}+y_{n}\left(x ; \tilde{\mu}_{t}\right)^{2}\right)^{2}}\right. \\
& \left.+\left(\int_{\left[-N_{n}, N_{n}\right]} \frac{(u-x) \tilde{\sigma}_{t}(d u)}{\left((u-x)^{2}+y_{n}\left(x ; \tilde{\mu}_{t}\right)^{2}\right)^{2}}\right)^{2} / \int_{\left[-N_{n}, N_{n}\right]} \frac{\tilde{\sigma}_{t}(d u)}{\left((u-x)^{2}+y_{n}\left(x ; \tilde{\mu}_{t}\right)^{2}\right)^{2}}\right) .
\end{aligned}
$$


We easily conclude from (5.17) that

$$
c_{7}(\mu) \leq f_{t}^{\prime}(x) \leq c_{8}(\mu), \quad|x|<M_{n}\left(\tilde{\mu}_{t}\right) .
$$

Fix $s \in \mathbb{R}$ and real $x$ and $\tilde{x}$ such that $s=f(x)=f_{t}(\tilde{x})$. Denote as well $\tilde{s}=f_{t}(x)$.

We see that

$$
\begin{aligned}
s-\tilde{s} & =f(x)-f_{t}(x)=Z_{n}^{(-1)}\left(x+i y_{n}(x ; \tilde{\mu})\right)-W_{n}^{(-1)}\left(x+i y_{n}\left(x ; \tilde{\mu}_{t}\right)\right) \\
& =Z_{n}^{(-1)}\left(x+i y_{n}(x ; \tilde{\mu})\right)-W_{n}^{(-1)}\left(x+i y_{n}(x ; \tilde{\mu})\right) \\
& +W_{n}^{(-1)}\left(x+i y_{n}(x ; \tilde{\mu})\right)-W_{n}^{(-1)}\left(x+i y_{n}\left(x ; \tilde{\mu}_{t}\right)\right) .
\end{aligned}
$$

Since, for $z_{1}, z_{2} \in \mathbb{C}^{+}$,

$$
W_{n}^{(-1)}\left(z_{1}\right)-W_{n}^{(-1)}\left(z_{2}\right)=z_{1}-z_{2}+(n-1) \int_{\left[-N_{n}, N_{n}\right]} \frac{\left(z_{2}-z_{1}\right) \tilde{\sigma}_{t}(d u)}{\left(u-z_{1}\right)\left(u-z_{2}\right)},
$$

we obtain the upper bound, for $\left|z_{1}\right| \geq 2 N_{n}$ and $\left|z_{2}\right| \geq 2 N_{n}$,

$$
\left|W_{n}^{(-1)}\left(z_{1}\right)-W_{n}^{(-1)}\left(z_{2}\right)\right| \leq c(\mu)\left|z_{1}-z_{2}\right| .
$$

Therefore we have

$$
\left|W_{n}^{(-1)}\left(x+i y_{n}(x ; \tilde{\mu})\right)-W_{n}^{(-1)}\left(x+i y_{n}\left(x ; \tilde{\mu}_{t}\right)\right)\right| \leq c(\mu)\left|y_{n}(x ; \tilde{\mu})-y_{n}\left(x ; \tilde{\mu}_{t}\right)\right|
$$

and, by Lemma 5.8, we get finally

$$
\left|W_{n}^{(-1)}\left(x+i y_{n}(x ; \tilde{\mu})\right)-W_{n}^{(-1)}\left(x+i y_{n}\left(x ; \tilde{\mu}_{t}\right)\right)\right| \leq c(\mu) e^{-N_{n} /\left(200 L\left(N_{n}\right)\right)} .
$$

By Lemma 5.6, we obtain as well

$$
\left|Z_{n}^{(-1)}\left(x+i y_{n}(x ; \tilde{\mu})\right)-W_{n}^{(-1)}\left(x+i y_{n}(x ; \tilde{\mu})\right)\right| \leq c(\mu) e^{-N_{n} /\left(200 L\left(N_{n}\right)\right)} .
$$

Therefore, applying (5.20) and (5.21) to (5.19), we arrive at the desired estimate

$$
\left|f_{t}(x)-f_{t}(\tilde{x})\right|=|s-\tilde{s}| \leq c(\mu) e^{-N_{n} /\left(200 L\left(N_{n}\right)\right)} .
$$

Since $f_{t}(x)-f_{t}(\tilde{x})=f_{t}^{\prime}(x+\theta(\tilde{x}-x))(\tilde{x}-x)$, we have in view of (5.18) $|\tilde{x}-x| \leq$ $c(\mu) e^{-N_{n} /\left(100 L\left(N_{n}\right)\right)} \mid$.

Now we estimate $\left|y_{n}\left(x ; \tilde{\mu}_{t}\right)^{2}-y_{n}\left(\tilde{x} ; \tilde{\mu}_{t}\right)^{2}\right|$, using the formula

$$
y_{n}\left(x ; \tilde{\mu}_{t}\right)^{2}-y_{n}\left(\tilde{x} ; \tilde{\mu}_{t}\right)^{2}=\left.\left(y_{n}\left(x ; \tilde{\mu}_{t}\right)^{2}\right)^{\prime}\right|_{x=x^{*}}(\tilde{x}-x),
$$

where $x^{*}=x+\theta(\tilde{x}-x)$. By Lemma 5.9, we see that $\left|\left(y_{n}\left(x ; \tilde{\mu}_{t}\right)^{2}\right)^{\prime}\right| \leq c(\mu) n^{1 / 2}$ for $|x|<$ $M_{n}\left(\tilde{\mu}_{t}\right)$. If $y_{n}\left(x ; \tilde{\mu}_{t}\right)+y_{n}\left(\tilde{x} ; \tilde{\mu}_{t}\right) \geq e^{-N_{n} /\left(400 L\left(N_{n}\right)\right)}$, we obtain from (5.23) that $\mid y_{n}\left(x ; \tilde{\mu}_{t}\right)-$ $y_{n}\left(\tilde{x} ; \tilde{\mu}_{t}\right) \mid \leq c(\mu) e^{-N_{n} /\left(400 L\left(N_{n}\right)\right)}$. The same bound holds of cause in the case $y_{n}\left(x ; \tilde{\mu}_{t}\right)+$ $y_{n}\left(\tilde{x} ; \tilde{\mu}_{t}\right)<e^{-N_{n} /\left(400 L\left(N_{n}\right)\right)}$.

Since $\left|x+i y_{n}\left(x ; \tilde{\mu}_{0}\right)-\tilde{x}-i y_{n}\left(\tilde{x} ; \tilde{\mu}_{t}\right)\right| \leq|x-\tilde{x}|+\left|y_{n}\left(x ; \tilde{\mu}_{0}\right)-y_{n}\left(x ; \tilde{\mu}_{t}\right)\right|+\left|y_{n}\left(x ; \tilde{\mu}_{t}\right)-y_{n}\left(\tilde{x} ; \tilde{\mu}_{t}\right)\right|$, we finally conclude that

$$
\left|x+i y_{n}\left(x ; \tilde{\mu}_{0}\right)-\tilde{x}-i y_{n}\left(\tilde{x} ; \tilde{\mu}_{t}\right)\right| \leq c(\mu) e^{-N_{n} /\left(400 L\left(N_{n}\right)\right)}
$$


and we have the estimate $\left|Z_{n}(s)-W_{n}(s)\right| \leq c(\mu) e^{-N_{n} /\left(400 L\left(N_{n}\right)\right)}$. Lemma 5.5 is proved.

Now we can estimate $\Delta\left(\tilde{\mu}^{n \boxplus}, \tilde{\mu}_{t}^{n \boxplus}\right)$ using the inversion formula. Recalling that $\tilde{\mu}^{n \boxplus}$ and $\tilde{\mu}_{t}{ }^{n \boxplus}$ are supported on the segment $\left[-c(\mu)(n-1) N_{n}, c(\mu)(n-1) N_{n}\right]$, we have, for $x$ from this segment,

$$
\begin{aligned}
\tilde{\mu}^{n \boxplus}\left(\left(-c(\mu)(n-1) N_{n}, x\right)\right) & -\tilde{\mu}_{t}^{n \boxplus}\left(\left[-c(\mu)(n-1) N_{n}, x\right)\right) \\
& =-\lim _{\eta \rightarrow 0} \frac{1}{\pi} \int_{\left[-c(\mu)(n-1) N_{n}, x\right)} \Im\left(G_{\tilde{\mu}}\left(\tilde{Z}_{n}(u+i \eta)\right)-G_{\tilde{\mu}_{t}}\left(\tilde{W}_{n}(u+i \eta)\right) d u .\right.
\end{aligned}
$$

Applying to this formula estimate (5.9) and (5.10), we get the upper bound

$$
\Delta\left(\tilde{\mu}^{n \boxplus}, \tilde{\mu}_{t}^{n \boxplus}\right) \leq c(\mu) n N_{n} e^{-c(\mu) N_{n} / L\left(N_{n}\right)} .
$$

The statement of the theorem follows immediately from (5.7) and (5.24).

\section{Probability measures $\mu$ With PoWer DeCRease in $\Delta\left(\mu^{n \boxplus}, \mathbf{D}^{\boxplus}\right)$}

As the first step we prove the following result.

Theorem 6.1. Let $\mu$ and $\rho$ belong to class $\mathcal{M}_{2 k}$, where $k \in \mathbb{N}_{1}$, and let $m_{j}(\mu)=$ $m_{j}(\rho), j=1, \ldots, 2 k-1$. Then

$$
\Delta\left(\mu^{n \boxplus}, \rho^{n \boxplus}\right) \leq c(\mu, \rho) n^{-(k-4) / 4}, \quad n \in \mathbb{N} .
$$

We prove this theorem, using arguments of Section 5.

Proof. Introduce the segment $\left[-N_{n}, N_{n}\right]$ with $N_{n}:=\sqrt{n} / \tilde{c}(\mu, \rho)$ with sufficiently large constant $\tilde{c}(\mu, \rho)>0$ and consider truncated on this segment probability measures $\tilde{\mu}$ and $\tilde{\rho}$. By the bounds

$$
\mu(\{|x| \geq N\}) \leq \frac{m_{2 k}(\mu)}{N^{2 k}}, \quad \mu(\{|x| \geq N\}) \leq \frac{m_{2 k}(\rho)}{N^{2 k}}
$$

for $N \geq 1$, and Proposition $\underline{3.5}$, we have

$$
\Delta\left(\rho^{n \boxplus}, \mu^{n \boxplus}\right) \leq \Delta\left(\tilde{\rho}^{n \boxplus}, \tilde{\mu}^{n \boxplus}\right)+c(\mu, \rho) N_{n}^{-2 k+1}, \quad n \in \mathbb{N},
$$

where $c(\mu, \rho)>0$ does not depend on $\tilde{c}(\mu, \rho)$. In the sequel constants $c(\mu, \rho)$ do not depend on $\tilde{c}(\mu, \rho)$ as well.

As before in Section $5 G_{\tilde{\mu}^{n \boxplus}}(z)=G_{\tilde{\mu}}\left(\tilde{Z}_{n}(z)\right)$ and $G_{\tilde{\rho}^{n \boxplus}}(z)=G_{\tilde{\rho}}\left(\tilde{W}_{n}(z)\right)$, where $\tilde{Z}_{n}(z)$ and $\tilde{W}_{n}(z)$ are belonging to the class $\mathcal{F}$ and satisfy the functional equations (4.21) and (4.23) with $\tilde{\rho_{n}}=\tilde{\rho}$, respectively. By Lemma 3.9 , we conclude that $\tilde{Z}_{n}(z)$ and $\tilde{W}_{n}(z)$ admit representations (4.22) and (4.24), respectively, where the measures $\tilde{\sigma}_{n}$ and $\tilde{\tau}_{n}$ satisfy the conditions, for some $c(\mu, \rho)>0$,

$$
\begin{aligned}
& \tilde{\sigma}_{n}(\mathbb{R})=\tilde{\sigma}_{n}\left(\left[-c(\mu, \rho)(n-1) N_{n}, c(\mu, \rho)(n-1) N_{n}\right]\right), \\
& \tilde{\tau}_{n}(\mathbb{R})=\tilde{\tau}_{n}\left(\left[-c(\mu, \rho)(n-1) N_{n}, c(\mu, \rho)(n-1) N_{n}\right]\right) .
\end{aligned}
$$


As in Section 5 we can show that the probability measures $\tilde{\mu}^{n \boxplus}$ and $\tilde{\rho}^{n \boxplus}$ are supported on the segment $\left[-c(\mu, \rho)(n-1) N_{n}, c(\mu, \rho)(n-1) N_{n}\right]$.

As in Section 5 we easily obtain the analogue of (5.10)

$$
\left|G_{\tilde{\mu}}\left(\tilde{Z}_{n}(z)\right)-G_{\tilde{\rho}}\left(\tilde{W}_{n}(z)\right)\right| \leq \frac{c(\mu, \rho)}{N_{n}^{2}}\left|\tilde{Z}_{n}(z)-\tilde{W}_{n}(z)\right|, \quad z \in \mathbb{R} .
$$

Now we need to obtain an analogue of Lemma 5.5.

Lemma 6.2. The following upper bounds hold

$$
\left|\tilde{Z}_{n}(z)-\tilde{W}_{n}(z)\right| \leq \frac{c(\mu, \rho)}{n^{(k-2) / 4}}, \quad \Im z \geq 0 .
$$

In order to prove this lemma we need some auxiliary results which are analogues of Lemmas 5.6, 5.7.

Lemma 6.3. The upper bound holds, for $z \in \mathbb{C}^{+},|z| \geq 2 N_{n}$,

$$
\left|\tilde{Z}_{n}^{(-1)}(z)-\tilde{W}_{n}^{(-1)}(z)\right| \leq \frac{c(\mu, \rho)(n-1)}{N_{n}^{2 k-1}} .
$$

Lemma 6.4. The upper bound holds, for $z \in \mathbb{C}^{+},|z| \geq 2 N_{n}$,

$$
\frac{1}{\Im z}\left|\Im\left(\frac{1}{G_{\tilde{\mu}}(z)}-\frac{1}{G_{\tilde{\rho}}(z)}\right)\right| \leq \frac{c(\mu, \rho)}{N_{n}^{2 k-1}} .
$$

The proof of these two lemmas we obtain in the way as the proof of Lemmas 5.6,5.6.

Furthermore we repeat the argument of Section 5, replacing the measures $\tilde{\mu}$ and $\tilde{\rho}$ by the measures of $\tilde{\mu}$ and $\tilde{\mu}_{t}$ and the functions $y_{n}(x ; \tilde{\mu}), y_{n}(x ; \tilde{\rho})$ by $y_{n}(x ; \tilde{\mu}), y_{n}\left(x ; \tilde{\mu_{t}}\right)$. We use in these arguments the constants $c(\mu, \rho)$ instead of $c(\mu)$.

In the same way as in the proof of Lemma 5.8 we prove

Lemma 6.5. The following bound holds

$$
\left|y_{n}(x ; \tilde{\mu})-y_{n}(x ; \tilde{\rho})\right| \leq \frac{c(\mu, \rho)}{N_{n}^{(2 k-1) / 2}} .
$$

Using Lemmas 6.3, 6.5 we prove Lemma 6.2 in the way as in Section 5 we proved Lemma 5.5.

Now we estimate $\Delta\left(\tilde{\mu}^{n \boxplus}, \tilde{\rho}^{n \boxplus}\right)$ using the inversion formula. Recalling that $\tilde{\mu}^{n \boxplus}$ and $\tilde{\rho}^{n \boxplus}$ are supported on the segment $\left[-c(\mu, \rho)(n-1) N_{n}, c(\mu, \rho)(n-1) N_{n}\right]$, we have, for $x$ from this segment,

$$
\begin{aligned}
\tilde{\mu}^{n \boxplus}\left(\left(-c(\mu, \rho)(n-1) N_{n}, x\right)\right) & -\tilde{\rho}^{n \boxplus}\left(\left[-c(\mu)(n-1) N_{n}, x\right)\right) \\
& =-\lim _{\eta \rightarrow 0} \frac{1}{\pi} \int_{\left[-c(\mu, \rho)(n-1) N_{n}, x\right)} \Im\left(G_{\tilde{\mu}}\left(\tilde{Z}_{n}(u+i \eta)\right)-G_{\tilde{\rho}}\left(\tilde{W}_{n}(u+i \eta)\right) d u .\right.
\end{aligned}
$$


Applying to this formula the estimates (6.2) and (6.3), we get the upper bound

$$
\Delta\left(\tilde{\mu}^{n \boxplus}, \tilde{\rho}^{n \boxplus}\right) \leq c(\mu, \rho) n N_{n} \frac{1}{N_{n}^{2}} \frac{1}{n^{(k-2) / 4}} \leq \frac{c(\mu \cdot \rho)}{n^{(k-4) / 4}} .
$$

The statement of the theorem follows immediately from (6.1) and (6.5).

As the second step we prove the following result, using arguments of Section 4.

Theorem 6.6. Let $\mu \in \mathcal{M}_{2 k}$, where $k \in \mathbb{N}_{1}$, and let $\alpha_{2 k}(\mu)<0$. Let there exist $a \boxplus$ infinitely divisible probability measure $\rho$ such that $m_{2 k}(\rho)<\infty$ and $m_{j}(\mu)=m_{j}(\rho), j=$ $1, \ldots, 2 k-1$. Then

$$
\Delta\left(\mu^{n \boxplus}, \rho^{n \boxplus}\right) \geq c(\mu, \rho) n^{-\frac{k^{2}+3 k+1}{k-1}}, \quad n \in \mathbb{N} .
$$

Proof. Assume that (6.6) does not hold. Then there exists a subsequence of increasing positive integers $\left.n_{l}\right\}_{l=1}^{\infty}$ such that

$$
\Delta\left(\mu^{n_{l} \boxplus}, \rho^{n_{l} \boxplus}\right) \leq \varepsilon_{n_{l}}:=o(1) n_{l}^{-\frac{k^{2}+3 k+1}{k-1}}, \quad n_{l} \in \mathbb{N} .
$$

Our nearest aim is to show that (6.7) implies $m_{2 k}(\mu)=m_{2 k}(\rho)$.

In the sequel we denote $n_{l}$ by $n$. As in Section 4 we denote by $\tilde{\mu}$ and $\tilde{\rho}$ the truncated probability measures of $\mu$ and $\rho$, respectively, with the parameter $N_{n}=\left(n / \varepsilon_{n}\right)^{1 /(2 k)}$. We denote in this section $\varepsilon_{n}^{*}=\varepsilon_{n}+n / N_{n}^{2 k}$. Instead of Lemma 4.3 we will use the estimate $\rho\left(|u|>2 N_{n}\right) \leq m_{2 k}(\rho) /\left(2 N_{n}\right)^{2 k}$. Then repeating the argument of Section 4 we arrive at the following upper bound, for every $v>0$,

$$
\int_{[0, v]}|\varphi(t ; \tilde{\mu})-\varphi(t ; \tilde{\rho})| d t \leq(c(\mu)+c(\rho)) n^{5 / 2} N_{n}^{2} e^{v h_{n}} \varepsilon_{n}^{*} .
$$

Since

$$
\begin{aligned}
\varphi(t ; \tilde{\mu})-\varphi(t ; \tilde{\rho}) & =\varphi(t ; \mu)-\varphi(t ; \rho)+\mu\left(|u|>2 N_{n}\right)-\rho\left(|u|>2 N_{n}\right) \\
& -\int_{|u|>2 N_{n}} e^{i t u} \mu(d u)+\int_{|u|>2 N_{n}} e^{i t u} \rho(d u), \quad t \in \mathbb{R},
\end{aligned}
$$

we easily obtain

$$
\begin{aligned}
\left|\int_{[0, v]}(\varphi(t ; \mu)-\varphi(t ; \rho)) d t\right| & \leq \int_{[0, v]}|\varphi(t ; \tilde{\mu})-\varphi(t ; \tilde{\rho})| d t+\frac{m_{2 k}(\mu)+m_{2 k}(\rho)}{\left(2 N_{n}\right)^{2 k}} \\
& \leq(c(\mu)+c(\rho)) n^{5 / 2} N_{n}^{2} e^{v h_{n}} \varepsilon_{n}^{*} .
\end{aligned}
$$

By Taylor's formula

$$
\varphi(t ; \mu)-\varphi(t ; \rho)=\frac{m_{2 k}(\mu)-m_{2 k}(\rho)}{2 k !} t^{2 k}+o\left(t^{2 k}\right)
$$

for small $t>0$, and we obtain from the preceding inequality with $v=1 / \sqrt{n}$ the relation $m_{2 k}(\mu)-m_{2 k}(\rho)=o(1)$ as $n \rightarrow \infty$. Thus, we proved that $m_{2 k}(\mu)=m_{2 k}(\rho)$. It remains to note that then $\alpha_{j}(\mu)=\alpha_{j}(\rho)$ for $j=1, \ldots, 2 k$. Since $\alpha_{2 k}(\rho) \geq 0$, we arrive at the contradiction. Hence (6.6) is proved. 
Theorem 2.10 follows from Theorems 6.1 and 6.6.

\section{REFERENCES}

[1] Akhiezer, N. I. The classical moment problem and some related questions in analysis. Hafner, New York (1965).

[2] Arak, T. V. and Zaitsev A. Yu. Uniform limit theorems for sums of independent random variables. Proceedings of the Steklov institute of mathematics, Issue 1, (1988).

[3] Arak, T. V. On the rate of convergence in Kolmogorov's uniform limit theorem, I,II. (Russian) Teoreiya Veroyatn. i Ee Primenenie, 26, No. 2, 225-245 (1981); 36, No. 3, 449-463 (1981);.

[4] Arak, T. V. An improvement of the lower bound for the rate of convergence in Kolmogorov's uniform limit theorem. (Russian) Teoreiya Veroyatn. i Ee Primenenie, 27, No. 4, 767-772 (1982);

[5] Bercovici, H., and Belinschi, S, T. A new approach to subordination results in free probability. J. Anal. Math., 101, 357-365 (2007).

[6] Belinschi, S, T. The Lebesgue decomposition of the free additive convolutions of two probability distributions. Probab. Theory Relat. Fields,142, 125-150 (2008).

[7] Bercovici, H., and Voiculescu, D. Free convolution of measures with unbounded support. Indiana Univ. Math. J., 42, 733-773 (1993).

[8] Bercovici, H., and Pata, V. Stable laws and domains of attraction in free probability theory. Annals of Math., 149, 1023-1060, (1999).

[9] Biane, Ph. Processes with free increments. Math. Z., 143-174 (1998).

[10] Chistyakov, G. P. Bounds for approximations of $n$-fold convolutions of distributions with unlimited divisibility and the moment problem. Journal of Mathematical Sciences, 76, No. 4, 2493-2511 (1995).

[11] Chistyakov, G. P. and Götze, F. The arithmetic of distributions in free probability theory. Cent. Eur. J. Math., 9(5), 997-1050 (2011), DOI: 10.2478/sl 1533-011-0049-4, ArXiv:math./0508245

[12] Chistyakov, G. P. and Götze, F. Limit theorems in free probability theory, I. Ann. Probab., 36, 54-90 (2008).

[13] Chistyakov, G. P. and Götze, F. Asymptotic expansions in the CLT in free probability. Theory Related Fields, 157, no. 1-2,107-156 (2013).

[14] Chistyakov, G. P. and Götze, F. Free infinitely divisible approximations of n-fold free convolutions. Prokhorov and contemporary probability theory. Springer proceeding in mathematics and statistics, 33, 225-237 (2013).

[15] Doeblin, W. Sur les sommes d'un grand nombre de variables aléatoires indépendantes. Bull. Sci. Math. (2) 63, 23-32, 35-64 (1939)

[16] Gnedenko, B. V. and Kolmogorov, A. N. Limit distributions for sums of independent random variables Addison-Wesley Publishing Company, (1968).

[17] Hiai, F. and Petz, D. The semicircle law, free random variables and entropy. Math. Surveys Monogr., 77, Amer. Math. Soc., Providence, RI (2000).

[18] Kargin, V. On superconvergence of sums of free random variables. Ann. Probab., 35, 1931-1949 (2007)

[19] Kolmogorov, A. N. On some work of recent years in the area of limit theorems of probability theory. (Russian) Vestnik Moskov. Univ., No. 10 (Ser. Fiz-Mat. i Estestv. Nauk vyp. 7), 29-38 (1953).

[20] Kolmogorov, A. N. Two uniform limit theorems for sums of independent terms. (Russian) Teoreiya Veroyatn. i Ee Primenenie, 1, No. 4, 426-436 (1956). 
[21] Lamperti, J. Probability MMS, New York, Amsterdam (1966).

[22] Maassen, H. Addition of Freely Independent Random Variables. Journal of functional analysis, 106, 409-438 (1992).

[23] Nica, A., Speicher, R., Lectures on the Combinatorics of Free Probability. Lecture Note Series, No 335, Cambridge University Press (2006).

[24] Prokhorov, Yu. V. On sums of identically distributed variables. (Russian) Dokl. Akad. Nauk SSSR, 105, No. 4, 645-647 (1955).

[25] Seneta, E. Regularly Varying Functions. Lecture Notes in Mathematics, Springer-Verlag, Berlin . Heidelberg · New York (1976).

[26] Speicher, R., Combinatorical theory of the free product with amalgamation and operator-valued free probability theory. Mem. A.M.S., 627 (1998).

[27] Takasaki, H. Theory of Operetor Algebras II. Springer-Verlag, Berlin · Heidelberg · New York (2003).

[28] Voiculescu, D.V. Addition of certain non commuting random variables. J. Funct. Anal., 66, 323-346 (1986).

[29] Voiculesku, D., Dykema, K., and Nica, A. Free random variables. CRM Monograph Series, No 1, A.M.S., Providence, RI (1992).

[30] Voiculescu, D.V. The analogues of entropy and Fischer's information measure in free probability theory. I. Comm. Math. Phys., 155, 71-92 (1993).

Gennadil Chistyakov

FACULTÄT FÜR MATHEMATIK

UNIVERSITÄT BIELEFELD

POSTFACH 100131

33501 BIELEFELD

Germany

Email address: chistyak@mathematik.uni-bielefeld.de

FRIEDRICH GÖTZE

FAKULTÄT FÜR MATHEMATIK

UNIVERSITÄT BIELEFELD

POSTFACH 100131

33501 BIELEFELD

Germany

Email address: goetze@mathematik.uni-bielefeld.de 